\title{
TOWARD AN INTEGRATED MODEL OF PATHOLOGICAL PERSONALITY TRAITS: COMMON HIERARCHICAL STRUCTURE OF THE PID-5 AND THE DAPP-BQ
}

\author{
Fernando Gutiérrez, PhD, José Ruiz, PhD, Josep Maria Peri, PhD, \\ Miguel Gárriz, PhD, Gemma Vall, PhD, \\ and Myriam Cavero, $\mathrm{MD}, \mathrm{PhD}$
}

\begin{abstract}
A dimensional classification seems to be the next move in the personality disorders field. However, it is not clear whether there is one dimensional model or many, or whether the currently available dimensional instruments measure the same traits. To help clarify these issues, the authors administered the Personality Inventory for DSM-5 (PID-5) and the Dimensional Assessment of Personality Pathology (DAPP-BQ) to 414 psychiatric outpatients. Factor analyses showed that a common hierarchical structure underlies both instruments, even if each one measures slightly different aspects of it. Disattenuated correlations indicated that, at the lower order level, two thirds of the PID-5 and DAPP-BQ facets measure essentially the same traits, although the pairings were not exactly as predicted. Among higher order domains, only PID Negative Affectivity and Detachment converged unambiguously with DAPP Emotional Dysregulation and Inhibition. Overall, the PID-5 and the DAPP-BQ reflect, with small divergences, one and the same structure of pathological personality traits.
\end{abstract}

Keywords: personality disorders, classification, dimensional model, PID5, DAPP-BQ, hierarchical factor analysis

\footnotetext{
Supplemental materials are available online.

From Personality Disorder Unit, Institute of Neuroscience, Hospital Clínic de Barcelona, Spain, and Institut d'Investigacions Biomèdiques August Pi Sunyer (IDIBAPS), Barcelona, Spain (F. G.); Department of Clinical Psychology and Psychobiology, Section of Personality, Assessment and Psychological Treatments, University of Barcelona, Spain (J. R.); Institute of Neuroscience, Hospital Clínic de Barcelona, Spain (J. M. P.); CSMA La Mina, Institut de Neuropsiquiatria i Addiccions, Parc de Salut Mar, Barcelona, Spain (M. G.); Department of Psychiatry, Mental Health, and Addiction, GSS-Hospital Santa Maria, Lleida, and IRB (Institut de Recerca Biomèdica), Lleida, Spain (G. V.); and Institute of Neuroscience, Hospital Clínic de Barcelona, Spain (M. C.).

This work was supported by project PI15/00536, integrated in the Plan Estatal de Investigación Cientifica y Técnica y de Innovación 2013-2016, and cofinanced by the ISCIII-Subdirección General de Evaluación and the European Regional Development Fund (ERDF) (PI: F. Gutiérrez).

Fernando Gutiérrez, José Ruiz, Josep Maria Peri, Miguel Gárriz, Gemma Vall, and Myriam Cavero declare that they have no conflict of interest.

Address correspondence to Fernando Gutiérrez, Personality Disorder Unit, Institute of Neuroscience, Hospital Clinic de Barcelona, Villarroel 170, 08036 Barcelona, Spain. E-mail fguti@clinic.ub.es
} 
We are irrevocably moving toward a dimensional taxonomy of personality disorders. The theoretical and empirical reasons for this change are plentiful and have been profusely outlined elsewhere (Ofrat, Krueger, \& Clark, 2018; Widiger, 2018). Moreover, there are strong grounds to believe that this taxonomy essentially consists of a set of five universal dimensions-Negative Affect, Asociality, Disinhibition, Antagonism, Psychoticism-and that most current instruments reflect these basic dimensions to a considerable extent (Clark et al., 2018; Krueger et al., 2011; Markon, Krueger, \& Watson, 2005; Tyrer et al., 2011; Widiger \& Simonsen, 2005).

However, although agreement on a classificatory system is indeed imperative for the normal functioning of clinical practice and research (Widiger, 2018), this agreement may not be as complete as it should be. For example, there is no coincidence in the number of key personality domains. The models fully in force in the past two decades have proposed five pathological domains (Harkness, Finn, McNulty, \& Shields, 2012; Krueger, Derringer, Markon, Watson, \& Skodol, 2012; Tyrer et al., 2011), but also three (Clark, 1993), four (Austin \& Deary, 2000; Livesley \& Jackson, 2009; Mulder \& Joyce, 1997), six (Krueger et al., 2011), or seven (Cloninger, 2000). Factor analyses of current instruments, either alone or in combination, have also found a wide range of plausible solutions featuring from three to eight factors (Mulder, Newton-Howes, Crawford, \& Tyrer, 2011). Beyond the number, the discrepancies also concern the nature of key personality domains: For instance, compulsivity is an independent domain in the ICD-11 proposal but not in the DSM-5, whereas psychoticism exists in the DSM-5 but not in the ICD-11 (American Psychiatric Association, 2013; Tyrer et al., 2011). And central domains such as disinhibition, antagonism, and psychoticism do not always emerge from factor analyses, but they are sometimes replaced by unfamiliar factors such as affective dyscontrol, attention seeking, or subordination (Morey, Krueger, \& Skodol, 2013; Muñoz-Champel, Gutiérrez, Peri, \& Torrubia, 2018; Van den Broeck et al., 2014). Official classifications have faithfully reflected these vacillations. The DSM-5 workgroup proposed six domains of personality pathology in the first place (Krueger et al., 2011), but removed compulsivity shortly afterward (Krueger et al., 2012). The ICD11 contributors started with four broad dimensions (Mulder et al., 2011), moved in rapid succession to a five-domain system and then to a different one (Tyrer et al., 2011; Tyrer, Reed, \& Crawford, 2015), and finally settled on six domains that recover borderline personality (Reed, 2018). Together with the concerns expressed by the boards of scientific societies (Herpertz et al., 2017; see diverging positions in Hopwood et al., 2018), this attests to the fact that no consensus on a single dimensional taxonomy has yet been reached (Widiger, 2018).

On the other hand, the fact that a common structure-such as the Big Four or the Big Five-underlies two instruments does not necessarily mean that they measure exactly the same traits, or that they are interchangeable. To give an example, PID-5 Antagonism (Personality Inventory for the DSM5; Krueger et al., 2012) and PSY-5 Aggressiveness (Personality Pathology Five; Harkness et al., 2012) have proved to load on the same factor, but they are by no means the same $(r=.44$; Anderson et al., 2013). In this respect, 
higher order personality domains have not shown as strong a convergence between instruments as they would if they were one and the same thing (Anderson et al., 2013; Watson, Stasik, Ro, \& Clark, 2013). And the evidence is still weaker at the facet level: Although the only published study confirmed 22 out of 24 predicted associations between the DAPP-BQ (Dimensional Assessment of Personality Pathology-Basic Questionnaire; Livesley \& Jackson, 2009) and the SNAP (Schedule for Nonadaptive and Adaptive Personality; Clark, 1993), correlations were moderate at best (mean $r=.53$, range .29 to .78; Clark, Livesley, Schroeder, \& Irish, 1996).

In sum, we have around a dozen empirically based measures of maladaptive personality traits that are becoming the diagnostic standard (Clark et al., 2018), but we still need to know whether each one represents a different model of pathological traits or whether they are all slightly differing reflections of a common underlying structure. If the latter, we would like to know whether they cover different aspects of this common structure, or whether they measure exactly the same traits and therefore can be used interchangeably. In the current study, we focus on the relationships between the PID-5 and the DAPP-BQ. Both questionnaires were originally designed to cover the entire range of maladaptive personality traits as broadly as possible, and both have a suitable number of facets for factor analysis $(25$ and 18 , respectively). Moreover, although their use is widespread, their relationships are not well known, beyond partial reports of their intercorrelations (Bastiaens et al., 2016; Berghuis, Ingenhoven, van der Heijden, Rossi, \& Schotte, 2019) and a joint factor analysis in a small nonclinical sample (Van den Broeck et al., 2014).

Our main objective is to examine, in a clinical sample, whether a common hierarchical structure underlies the PID-5 and the DAPP-BQ, and to what extent each instrument gives adequate coverage to this structure. Furthermore, we want to know whether both instruments measure the same or different personality traits. This knowledge would help to articulate the future dimensional taxonomy of personality disorders on more solid grounds.

\section{METHOD}

\section{PARTICIPANTS}

The sample comprised 414 outpatients (58\% female), aged 17 to 64 years $(M=33.9, S D=11.3)$, with suspected personality problems, referred for assessment to the Personality Disorder Unit of a general university hospital. Psychopathology was assessed by two experienced clinicians (F.G. and J.M.P.) through the Dual Diagnosis Screening Interview (Mestre-Pintó, Domingo-Salvany, Martín-Santos, \& Torrens, 2014). About two thirds of the sample $(65.2 \%)$ were diagnosed with one or more DSM-IV Axis I disorders. Main diagnoses included mild to moderate affective disorders $(16.7 \%)$, anxiety disorders $(15.0 \%)$, trauma- and stress-related disorders $(9.7 \%)$, mixed depressive-anxious disorders $(7.5 \%)$, eating disorders $(4.1 \%)$, substancerelated disorders $(3.4 \%)$, and other disorders $(8.6 \%)$, each with a prevalence 
under 4\%. The remaining 34.8\% did not have an Axis I disorder. Personality pathology was not diagnosed categorically, but was assessed through the PID-5 and the DAPP-BQ. Outpatients covered the entire range from normal to severely disordered personality, thus ensuring maximum variability for factor analysis (descriptive statistics in Supplement Table S1). The study was approved by the ethics committee of the hospital, and all participants provided informed consent.

\section{INSTRUMENTS}

The Personality Inventory for DSM-5 (PID-5; Krueger et al., 2012) is a selfreport consisting of 220 items scored on a 4-point Likert-type scale ranging from 0 (strongly disagree) to 3 (strongly agree). Items group into 25 facets that are empirically clustered into five higher order personality pathology domains: Negative Affectivity, Detachment, Antagonism, Disinhibition, and Psychoticism. This study uses the Spanish version, which has shown suitable psychometric properties (F. Gutiérrez et al., 2017). In this sample, internal consistency coefficients (Cronbach's alphas) ranged from .81 to .95 (mean $.88)$.

The Dimensional Assessment of Personality Pathology-Basic Questionnaire (DAPP-BQ; Livesley \& Jackson, 2009) is a self-report consisting of 290 items scored on a 5-point Likert-type scale ranging from 1 (strongly disagree) to 5 (strongly agree). It measures 18 lower order facets that are structured into four higher order domains labeled as Emotional Dysregulation, Inhibition, Dissocial Behavior, and Compulsiveness. The Spanish version has previously demonstrated good psychometric properties (J. A. Gutiérrez-Zotes et al., 2008), and average alpha reliability of facets was .89 (range from .83 to $.94)$ in our sample.

\section{DATA ANALYSIS}

Descriptives for the PID-5 and the DAPP-BQ were obtained and converted into $T$ scores based on the respective community norms (F. Gutiérrez et al., 2017; J. A. Gutiérrez-Zotes et al., 2008). We first examined the joint hierarchical structure of the 25 PID-5 and 18 DAPP-BQ facets. A series of exploratory factor analyses (EFA) were performed following a bass-ackwards approach (Goldberg, 2006) with two modifications: In accordance with current recommendations (Osborne, 2014), we applied unweighted least squares (ULS) extraction instead of principal components (PC) because ULS is better suited for identifying latent variables, and we rotated to promax (kappa = 4) instead of varimax so as not to artificially constrain possible associations between factors. However, PC and varimax were performed in addition in order to compare methods. In the case of truly uncorrelated factors, orthogonal and oblique rotations would be identical. One to $k$ factors were successively retained, $k$ being the maximum number of psychologically interpretable factors with three loadings over .30 and acceptable fit. Regression-based factor scores were computed for all factors and correlated between contiguous levels of the hierarchy to obtain the path coefficients. The associations 
of PID-5 and DAPP-BQ domains with the factors were calculated to aid in interpreting the structure. Furthermore, the goodness-of-fit of each solution was tested through $\chi^{2}$ and complementary fit indices: namely, goodness-of-fit index (GFI), adjusted goodness-of-fit index (AGFI), and root mean square of residuals (RMSR) as absolute indices; comparative fit index (CFI) and nonnormed fit index (NNFI) as incremental indices; and the loading simplicity index (LS) (Hooper, Coughlan, \& Mullen, 2008; Lorenzo-Seva, 2003). GFI, AGFI, CFI, and NNFI indexes over .95, RMSR below .05, and LS the closest to 1 were considered a satisfactory fit. Finally, factor solutions were tested for congruence with the only previously published study (Van den Broeck et al., 2014) by means of Tucker's coefficients $(\phi)$, with .85 indicating fair similarity and .95 good similarity (Lorenzo-Seva \& ten Berge, 2006).

We then computed Pearson's correlations between the facets of both questionnaires. Correlations were disattenuated, that is, they were divided by the square root of the product of the respective alpha reliabilities: $r_{c}=r$ $/ \sqrt{ }\left(r_{\mathrm{xx}} \cdot r_{\mathrm{yy}}\right)$. This procedure estimates the "true" relationships between two variables once the effect of measurement error has been counted out (Osborne, 2003). Complementarily, because relationships between facets were not expected to be mostly biunivocal, we used multiple regression analysis to predict each PID-5 facet from the entire set of DAPP-BQ facets and vice versa. Adjusted $R^{2}$ coefficients were taken as indicators of the relative comprehensiveness of each questionnaire. For the sake of parsimony, increments in explanatory power of less than $\Delta R^{2}<.01$ were not considered. Disattenuation was not applied to regression coefficients because measurement error may distort them in different directions for each predictor (Williams, Gómez Grajales, \& Kurkiewicz, 2013). Residuals were analyzed through the Durbin-Watson test to determine their possible interdependence. Finally, through an item response theory (IRT) analysis using Samejima's Graded Response Model, we examined whether the PID-5 and the DAPP-BQ measure the same or different levels of severity of the resulting factors. IBM SPSS v.24, Factor v.10.3.1 (Lorenzo-Seva \& Ferrando, 2015), and R package "ltm" (Rizopoulos, 2006) were used for all analyses.

\section{RESULTS}

We first examined the joint hierarchical structure of the PID-5 and DAPP-BQ facets (Figure 1). From the top down, the hierarchy was headed by a general factor of Personality Pathology with loadings of over .30 for $86 \%$ of facets (full loadings in Supplement Table S2). From this construct, two broad factors emerged that were labeled Internalizing and Externalizing. The Internalizing factor split at the third level into two components labeled Detachment and Negative Affect, which remained virtually unchanged all the way down. The former component was marked by social withdrawal, intimacy avoidance, and restriction of affect, and accurately reproduced PID Detachment (mean disattenuated correlation $r_{c}=.94$ ) and DAPP Inhibition (.95). Negative Affect reflected a trend toward experiencing anxiety, depression, unstable humor, separation fears, and identity problems, which was close 


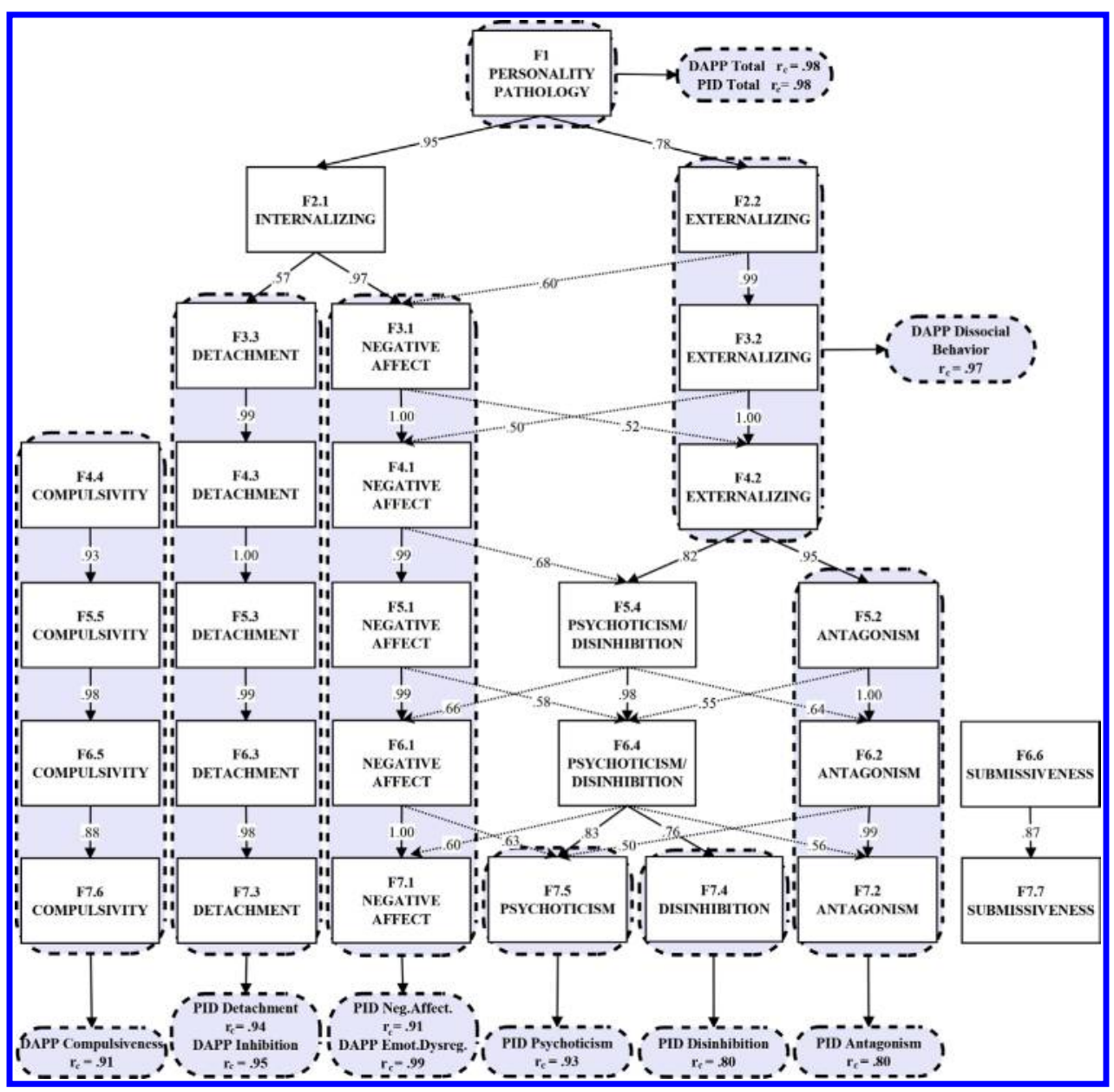

FIGURE 1. The Common Hierarchical Structure Underlying the PID5 and the DAPP-BQ. All between-level correlations $\geq .50$ are reported. Gray-shaded areas indicate the highest disattenuated correlations $\left(r_{c}\right)$ for each PID-5 and DAPP-BQ domain.

to PID Negative Affectivity (.91) and DAPP Emotional Dysregulation (.99). In the Externalizing branch, which globally corresponded to DAPP Dissocial Behavior (.97), an Antagonism component was severed at the fifth level, which included the use of manipulation and deception, attention seeking, arrogance, and heartlessness. The remaining factor was a compound that split further down into a Psychoticism factor encompassing unusual beliefs, perceptions, and behavior, and a Disinhibition factor defined by risk and stimulation seeking, impulsivity, and conduct problems. The latter three factors roughly reproduced the PID-5 domains of the same name $(.80, .93$, and .80, respectively). In addition, two narrower factors emerged that did not clearly come from any of the above constructs: Compulsivity rose at the fourth level, grouping together features of perfectionism, rigidity, and (reversed) irresponsibility, and was associated with DAPP Compulsiveness (.91); and Submissiveness emerged at the sixth level made up of the two homonymous 
TABLE 1. Promax-Rotated Seven-Factor Solution for the PID-5 and the DAPP-BQ

\begin{tabular}{|c|c|c|c|c|c|c|c|}
\hline & $\begin{array}{l}\text { Negative } \\
\text { Affect }\end{array}$ & Antagonism & Detachment I & Disinhibition I & Psychoticism & Compulsivity & Submissiveness \\
\hline DAPP Anxiousness & 1.01 & -.10 & .03 & .05 & -.14 & .17 & .11 \\
\hline PID Anxiousness & .97 & -.10 & -.10 & -.06 & -.11 & .13 & -.03 \\
\hline DAPP Identity Problems & .91 & -.04 & .25 & -.09 & -.12 & -.06 & -.03 \\
\hline PID Depressivity & .83 & -.10 & .19 & -.04 & .06 & -.07 & .05 \\
\hline PID Anhedonia & .77 & -.02 & .39 & -.17 & -.19 & -.11 & .02 \\
\hline $\begin{array}{l}\text { DAPP Insecure } \\
\text { Attachment }\end{array}$ & .76 & .09 & -.51 & -.11 & .12 & -.09 & .05 \\
\hline DAPP Submissiveness & .75 & -.02 & -.02 & -.08 & .13 & .02 & .56 \\
\hline $\begin{array}{l}\text { PID Separation Inse- } \\
\text { curity }\end{array}$ & .75 & .12 & -.47 & -.12 & .03 & -.14 & .11 \\
\hline DAPP Affective Lability & .72 & -.08 & -.12 & .31 & -.01 & .19 & -.29 \\
\hline DAPP Social Avoidance & .69 & .02 & .32 & -.20 & .04 & .04 & .17 \\
\hline PID Emotional Lability & .67 & -.15 & -.21 & .28 & .10 & .16 & -.13 \\
\hline PID Perseveration & .65 & .06 & .19 & .16 & -.06 & .26 & .13 \\
\hline DAPP Oppositionality & .64 & .04 & .11 & .34 & -.14 & -.21 & .18 \\
\hline PID Distractibility & .58 & -.04 & .12 & .33 & -.01 & -.17 & .16 \\
\hline PID Submissiveness & .57 & .05 & -.06 & -.19 & .14 & .03 & .55 \\
\hline PID Suspiciousness & .47 & .16 & -.02 & -.20 & .34 & -.08 & -.14 \\
\hline DAPP Suspiciousness & .44 & .21 & -.02 & -.17 & .35 & -.04 & -.14 \\
\hline DAPP Self-Harm & .40 & -.20 & .08 & .11 & .32 & .02 & -.01 \\
\hline PID Manipulativeness & -.22 & .85 & .02 & .15 & -.11 & .01 & .01 \\
\hline PID Deceitfulness & .01 & .80 & .06 & .06 & -.02 & -.08 & .16 \\
\hline DAPP Callousness & -.02 & .76 & .23 & .01 & -.03 & -.05 & -.11 \\
\hline PID Attention Seeking & .11 & .69 & -.25 & .16 & -.01 & .08 & .18 \\
\hline DAPP Rejection & .00 & .66 & -.01 & .17 & -.16 & .23 & -.24 \\
\hline DAPP Narcissism & .44 & .61 & -.24 & .01 & -.07 & .19 & .25 \\
\hline PID Grandiosity & -.26 & .61 & .03 & -.01 & .16 & .28 & .01 \\
\hline PID Callousness & -.14 & .57 & .30 & .06 & .09 & -.15 & -.24 \\
\hline $\begin{array}{l}\text { DAPP Intimacy } \\
\text { Problems }\end{array}$ & -.05 & -.08 & .80 & .00 & -.02 & .06 & -.11 \\
\hline PID Intimacy Avoidance & -.15 & -.02 & .74 & .08 & .02 & .07 & -.08 \\
\hline $\begin{array}{l}\text { DAPP Restricted } \\
\text { Expression }\end{array}$ & .18 & .07 & .71 & -.16 & .01 & .12 & .13 \\
\hline PID Withdrawal & .16 & -.04 & .71 & -.11 & .12 & .02 & -.07 \\
\hline $\begin{array}{l}\text { PID Restricted } \\
\text { Affectivity }\end{array}$ & -.12 & .33 & .65 & -.10 & .02 & .03 & .04 \\
\hline DAPP Stimulus Seeking & -.05 & 19 & -.16 & .71 & .16 & -.07 & -.10 \\
\hline PID Risk Taking & -.35 & .18 & -.07 & .60 & .20 & -.04 & -.13 \\
\hline PID Impulsivity & .22 & .08 & -.11 & .57 & .04 & -.12 & -.14 \\
\hline PID Irresponsibility & .29 & .22 & .16 & .39 & .02 & -.32 & .10 \\
\hline $\begin{array}{l}\text { DAPP Conduct } \\
\text { Problems }\end{array}$ & .02 & .25 & .12 & .38 & .15 & -.08 & -.18 \\
\hline $\begin{array}{l}\text { PID Unusual Beliefs \& } \\
\text { Experiences }\end{array}$ & -.11 & -.02 & -.01 & .21 & .87 & .10 & .09 \\
\hline $\begin{array}{l}\text { PID Perceptual } \\
\text { Dysregulation }\end{array}$ & .22 & -.07 & .08 & .22 & .70 & .05 & .18 \\
\hline $\begin{array}{l}\text { DAPP Cognitive } \\
\text { Distortion }\end{array}$ & 42 & -.15 & .09 & .19 & .60 & .01 & .16 \\
\hline PID Eccentricity & .21 & .04 & .28 & .17 & .43 & .10 & .05 \\
\hline PID Rigid Perfectionism & .23 & .15 & .19 & -.07 & -.01 & .80 & .01 \\
\hline DAPP Compulsivity & .03 & .07 & .08 & -.22 & .17 & .77 & .03 \\
\hline PID Hostility & .29 & .33 & .10 & .20 & -.07 & .02 & -.43 \\
\hline
\end{tabular}

Note. Factor loadings $\geq .30$ are in bold type; the highest loading for each facet is underlined.

PID-5 and DAPP-BQ facets plus (reversed) PID Hostility. Because factors were obliquely rotated, the full matrix of between- and within-level correlations is provided in Supplement Table S3 to aid interpretation. However, it is worth noting that an additional analysis using PC extraction and varimax rotation gave similar results, with congruence coefficients averaging $\phi=.96$ (range .89 to 1.00; Supplement Table S4). In contrast, the congruences of our 
TABLE 2. Goodness of Fit Statistics for the One- to Seven-Factor Solutions

\begin{tabular}{|c|c|c|c|c|c|c|c|}
\hline & 1 Factor & 2 Factors & 3 Factors & 4 Factors & 5 Factors & 6 Factors & 7 Factors \\
\hline Chi-square $\left(\chi^{2}\right)$ & $\begin{array}{c}(d f=860) \\
7894.71 \\
(p<.001)\end{array}$ & $\begin{array}{c}(d f=818) \\
6169.03 \\
(p<.001)\end{array}$ & $\begin{array}{c}(d f=777) \\
4563.07 \\
(p<.001)\end{array}$ & $\begin{array}{c}(d f=737) \\
3692.09 \\
(p<.001)\end{array}$ & $\begin{array}{c}(d f=698) \\
3111.53 \\
(p<.001)\end{array}$ & $\begin{array}{c}(d f=660) \\
2627.44 \\
(p<.001)\end{array}$ & $\begin{array}{c}(d f=623) \\
2288.84 \\
(p<.001)\end{array}$ \\
\hline \multicolumn{8}{|l|}{ Absolute Indices } \\
\hline $\begin{array}{l}\text { Goodness of Fit } \\
\text { Index (GFI) }\end{array}$ & .87 & .92 & .97 & .98 & .99 & .99 & .99 \\
\hline Adjusted GFI (AGFI) & .86 & .92 & .96 & .98 & .98 & .99 & .99 \\
\hline $\begin{array}{l}\text { GFI without diagonal } \\
\text { values }\end{array}$ & .84 & .91 & .96 & .98 & .99 & .99 & .99 \\
\hline $\begin{array}{l}\text { Adjusted GFI without } \\
\text { diagonal values }\end{array}$ & .84 & .90 & .95 & .97 & .98 & .99 & .99 \\
\hline $\begin{array}{l}\text { Root Mean Square of } \\
\text { Residuals (RMSR) }\end{array}$ & .13 & .10 & .07 & .05 & .04 & .04 & .03 \\
\hline \multicolumn{8}{|l|}{ Incremental Indices } \\
\hline $\begin{array}{l}\text { Comparative Fit } \\
\text { Index (CFI) }\end{array}$ & .44 & .57 & .70 & .76 & .81 & .84 & .87 \\
\hline $\begin{array}{l}\text { Non-Normed Fit } \\
\text { Index (NNFI) }\end{array}$ & .41 & .53 & .65 & .71 & .75 & .78 & .81 \\
\hline \multicolumn{8}{|l|}{ Simplicity } \\
\hline $\begin{array}{l}\text { Loading Simplicity } \\
\text { Index (LS) }\end{array}$ & - & $.33(\operatorname{Pc} 86)$ & .30 (Pc 98) & $.33(\operatorname{Pc} 100)$ & .37 (Pc 100) & $.37(\operatorname{Pc} 100)$ & $.37(\operatorname{Pc} 100)$ \\
\hline
\end{tabular}

two alternative solutions with a prior study (Van den Broeck et al., 2014) were poor, namely, .78 for the oblique rotation and .86 for the orthogonal rotation (Supplement Tables S2 and S4). The exceptions were the one-factor solution $(\phi=.98)$ and the Negative Affect factors of each level.

Procedures for determining the number of factors resulted in divergent solutions: Whereas the parallel test suggested five factors, Velicer's MAP suggested 11 factors (Supplement Table S5). Hierarchical analysis stopped at the seventh level (Table 1), which was the lowest one meeting our acceptability criteria: All factors were interpretable, had three loadings over .30, and provided the best fit $\left[\chi^{2}(623)=2288.84(p<.001)\right.$, AGFI $=.99$, RMSR $=.03, \mathrm{LS}=.37$ (Table 2)] . The seven-factor solution accounted for $71.1 \%$ of the common variance. On the premise that the shared structure of PID-5 and DAPP-BQ would be a good approximation to the universal structure of maladaptive personality traits, we examined the extent to which both instruments covered it adequately. Multiple regression analyses confirmed that they fared about equally well, and this was true for all dimensions: Negative Affect (PID-5 adjusted $R^{2}=.96$ vs. DAPP-BQ $=.98$ ), Antagonism (.95 vs. $.92)$, Detachment (.94 vs. .94), Disinhibition (.90 vs. .95), Psychoticism (.95 vs. .89), Compulsivity (.93 vs. .90), and Submissiveness (.84 vs. .92; all with $p<.001)$.

We then sought to ascertain whether the PID-5 and the DAPP-BQ measure the same traits. Disattenuated correlations suggested that some scales are identical between questionnaires (Table 3). Specifically, six PID-5 facets showed $r_{c}$ coefficients equal to or greater than .90 with five corresponding DAPP-BQ facets: PID with DAPP Suspiciousness, PID with DAPP Anxious- 


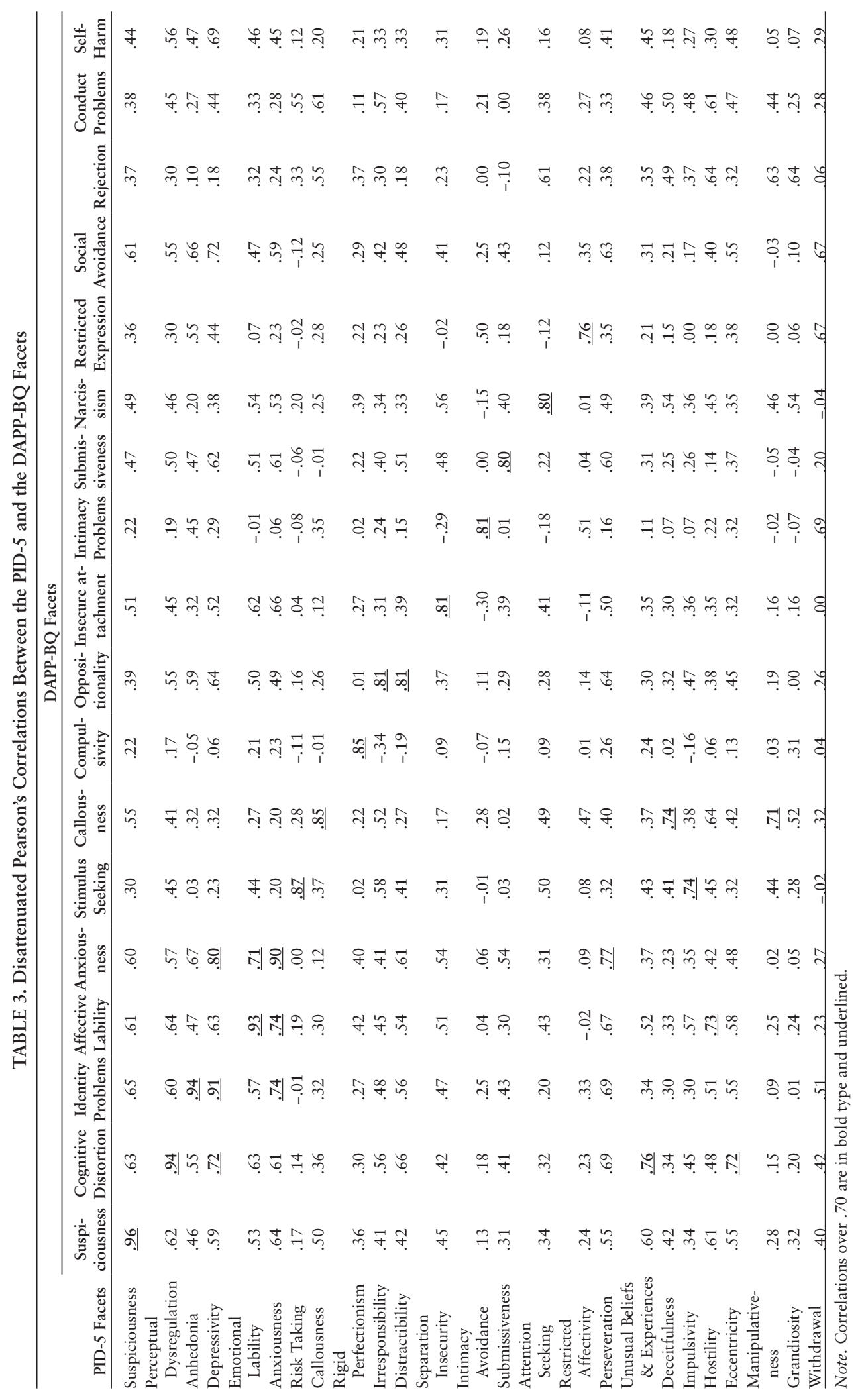


ness, PID Perceptual Dysregulation with DAPP Cognitive Distortion, PID Emotional Lability with DAPP Affective Lability, and PID Anhedonia and Depressivity with DAPP Identity Problems. Ten more PID-5 facets showed correlations equal to or greater than .80 with their corresponding DAPP-BQ facets, and nine correlated greater than .70 (an outline of the main disattenuated correlations is provided in Supplement Figure S1 and raw Pearson's correlations in Supplement Table S6). Only two PID-5 facets (Grandiosity and Withdrawal) and three DAPP-BQ facets (Rejection, Conduct Problems, and Self-Harm) lacked a clear image $\left(r_{c} \geq 70\right)$ in the other questionnaire. Furthermore, we also correlated PID-5 and DAPP-BQ higher order domains, finding a good correspondence of PID Negative Affectivity with DAPP Emotional Dysregulation $\left[r_{c}=.87(r=.83)\right]$ and PID Detachment with DAPP Inhibition $\left[r_{c}=.85(r=.77)\right]$. DAPP Dissocial Behavior was moderately associated with both PID Antagonism $\left[r_{c}=.75(r=.69)\right]$ and Disinhibition $\left[r_{c}=.62(r=.58)\right]$, and DAPP Compulsivity with the low pole of PID Disinhibition $\left[r_{c}=-.52\right.$ $(r=-.48)]$. These correlations were considered to form the monotrait-heteromethod diagonal of a multitrait multimethod matrix (Supplement Table S7). In this context, they proved to be higher [mean $\left.r_{c}=.72(r=.67)\right]$ than the heterotrait-monomethod and heterotrait-heteromethod blocks [mean $r_{\mathrm{c}}=$ $.32(r=.30)$ and $r_{c}=.27(r=.26)$, respectively], indicating good convergent and discriminant validity. However, the moderate correlation between PID Disinhibition and DAPP Emotional Dysregulation $\left[r_{c}=.66(r=.63)\right]$ was unexpected. PID Psychoticism showed an unspecific pattern of association because its true counterpart is not any DAPP-BQ domain but the Cognitive Distortion facet $\left[r_{c}=.88(r=.82)\right]$.

Because the correspondence between most PID-5 and DAPP-BQ facets was not biunivocal (nor was it expected to be so), we complementarily undertook multiple regression analyses, so that each facet in either questionnaire could be predicted by several facets from the other (Supplement Tables S8 and S9). Overall, the results confirmed the substantial overlap between the instruments, whether we predicted the PID-5 (mean explained variance $R^{2}=.59$, corresponding to $R=.77$ ) or the DAPP-BQ facets (mean $R^{2}=.66$, $R=.81$ ). The PID-5 was a slightly better predictor of the DAPP-BQ than vice versa, maybe because DAPP-BQ facets are fewer and often hierarchically superior. Another finding was that not all domains overlapped to the same extent. Facets belonging to PID Negative Affectivity-Emotional Lability, Anxiousness, Insecure Attachment-showed the highest explained variance (mean $R^{2}=.67$ ), followed by those belonging to Detachment (.61), Psychoticism (.58), Disinhibition (.56), and Antagonism (.43). The same was true of the DAPP-BQ facets belonging to Emotional Dysregulation (.71), Dissocial Behavior (.64), Compulsivity (.64), and Inhibition (.57).

Finally, an IRT analysis showed that both the DAPP-BQ and the PID-5 covered the more severe half of each factor better, DAPP Compulsivity being the only exception to this trend. Some divergences between the two instruments were also apparent. In the Negative Affect factor, the DAPP-BQ facets gave the most information at higher severity levels than the PID-5 facets (58.6\% vs. $51.9 \%$ on average). This difference was mainly due to DAPP Suspiciousness and Self-Harm peaking at the far right of the information curve 
(73.3\% and $82.5 \%$ respectively), whereas scales measuring anxiousness and lability on both questionnaires provided the most information at lower levels of the latent trait (complete test information curves in Supplement Figure S2). As for Antagonism, the DAPP-BQ facets were more informative at lower levels than the PID-5 facets (59.9\% vs. 70.4\%). PID Grandiosity $(80.1 \%)$ and Callousness $(82.5 \%)$ were especially balanced toward the upper end of the latent trait. However, these differences are not large, and they were negligible for all other factors: Detachment (55.9\% vs. $62.0 \%)$, Disinhibition $(57.0 \%$ vs. $57.9 \%)$, Psychoticism $(72.7 \%$ vs. $72.8 \%)$, Compulsivity $(49.3 \%$ vs. $45.7 \%)$, and Subordination $(59.1 \%$ vs. $57.5 \%)$.

\section{DISCUSSION}

In a sample of 414 outpatients, we examined whether a common structure underlies two comprehensive questionnaires of maladaptive personality traits, the PID-5 and the DAPP-BQ, and whether both questionnaires tap the same traits and are therefore interchangeable. Our results allow us to answer these questions in the affirmative, although some qualifications should be made.

Our starting point was as follows: If the PID-5 and the DAPP-BQ were based on independent attempts to measure the whole domain of maladaptive personality traits, their joint structure could well give a faithful reflection of the universal organization of that domain. We found that the two instruments indeed share a hierarchical structure whose similarity with the previous literature is notable: It includes a factor of general personality pathology at the top (Rushton, Irwing, \& Booth, 2010), the Big Two and Big Three immediately below (Markon et al., 2005), and the four or five essential dimensions of personality pathology from this level downwards: Negative Affect, Dissociality, Detachment, Psychoticism, and Compulsivity (Austin \& Deary, 2000; Markon et al., 2005; Mulder \& Joyce, 1997; Ofrat et al., 2018; Tyrer et al., 2011; Widiger \& Simonsen, 2005). Furthermore, both questionnaires proved excellent coverage of all the traits constituting the structure. This was true even of Psychoticism, although its absence as a higher order domain on the DAPP-BQ makes a difference.

In addition, two thirds of the PID-5 and DAPP-BQ facets are equivalent across instruments, as suggested by disattenuated correlations equal to or greater than .80 . This means that both questionnaires can be used almost interchangeably, using Supplement Figure S1 as guidance. Of course, this is not equally true for all traits. The overlap is greater for facets reflecting negative affect and is smaller for antagonism, with all other domains in between. For example, PID Grandiosity, Deceitfulness, and Manipulativeness, as well as DAPP Self-Harm and Conduct Problems, are exclusively held by their instruments and poorly represented in the other. Some other facets are well represented in the other questionnaire in terms of explained variance-PID Withdrawal, DAPP Rejection-but are spread over multiple facets that each account for a small part of variability. Quite often this is due to a DAPP-BQ facet-such as Anxiousness, Callousness, Cognitive Distortion...-encom- 
passing several PID-5 facets. A possible explanation is that the DAPP-BQ comes from the refactorization of an earlier 69-trait questionnaire (Livesley $\&$ Jackson, 2009), and some of their facets may have retained vestigial multidimensionality.

As for the associations between domains, they were similar to those previously reported in psychiatric patients (Bastiaens et al., 2016). We found only two clear equivalences: PID Negative Affectivity to DAPP Emotional Dysregulation, and PID Detachment to DAPP Inhibition. The externalizing branch was more problematic in this respect because domains are displaced relative to one another in regard to the level of abstraction. For example, DAPP Dissocial Behavior is superordinate to PID Antagonism and Disinhibition (Figure 1), and PID Psychoticism has its replica not in a DAPP-BQ domain, but in the Cognitive Distortion facet. Incidentally, it should be noted that DAPP Inhibition is not the opposite of PID Disinhibition $\left(r_{c}=.20\right)$ but the counterpart of PID Detachment (.85). This cautions against relying on trait labels.

Other issues at the domain level will need subsequent clarification. Compulsivity is sometimes the opposite pole of Disinhibition, as in the PID-5 and in Widiger and Simonsen (2005), and sometimes independent, as in the DAPPBQ and the ICD-11 (Tyrer et al., 2011). In our study, although the multitrait multimethod matrix ratified that PID Disinhibition is bipolar-with DAPP Dissocial Behavior at one pole and Compulsivity at the other-factor analysis did not find such a bipolar dimension at any level of the hierarchy, preventing any clear conclusion. The other knotty domain is Submissiveness, a narrow and infrequently reported factor that is, however, not residual: It emerged in our study before established domains such as Psychoticism and Disinhibition, and it has appeared repeatedly in previous works under labels such as Subordination, Need for Approval, or Anxious/Dependent (Clark et al., 1996; F. Gutiérrez, Vall, Peri, Gárriz, \& Garrido, 2014; Kushner, Quilty, Tackett, \& Bagby, 2011; Muñoz-Champel et al., 2018; Tyrer et al., 2011). This trait has been given less weight in the nosology than it deserves considering its alleged theoretical and clinical relevance (Johnson, Leedom, \& Muhtadie, 2012).

Some advice for future studies can be drawn from our results. On the one hand, relationships between dimensional models are harder to eyeball than they first appeared. If we take $r_{c} \geq .80$ as equivalence between facets, we were able to confirm only half of the 22 associations previously predicted by experts (Berghuis et al., 2019), and another five of our observed equivalences had not been foreseen. Similarly, the common assertion that all current personality instruments measure the same basic dimensions is challenged-or at least qualified-by the evidence of moderate and insufficiently specific associations between domains. Thus, we have to admit that grasping the lattice of relationships between traits requires more careful empirical examination than that carried out so far. On the other hand, hierarchical analyses are indispensable when it comes to reconciling discrepancies between models. Because domains are located at different levels of abstraction from one instrument to another, checking congruence "horizontally" can easily be misleading. In addition, analyses must go beyond the fifth level-in fact as far below as possible-because main factors do not always emerge at the outset. In our study, Psychoticism and Disinhibition did not emerge until the seventh level. 
In other studies, the dimensions that emerged late were Antagonism, Disinhibition, Subordination, or Compulsivity (F. Gutiérrez et al., 2014; Kushner et al., 2011; Morey et al., 2013), and Van den Broeck et al. (2014) did not find Psychoticism until the twelfth level.

This study has some weaknesses, and its limited sample size is the first of them. Even if 10 subjects per variable are usually considered sufficient in factor analysis, the requirements might in fact be higher for complex structures like personality (Osborne, 2014). Second, the statement that our hierarchy appears very similar to the literature is only tentative. In fact, at first glance our results also appeared similar to those of Van den Broeck et al. (2014), until Tucker's coefficients proved that they were not. This disagreement can only be partially attributed to the analytical method, because an alternative solution based on PC extraction and rotated orthogonally did not provide a much better match (Supplement Table S4). Maybe Van den Broeck et al.'s use of a small sample $(n=173)$ of nonclinical subjects would have restricted the range of pathological traits and alter the factorial solutions. Whatever the reason, it remains the case that mapping the relationships between models is still a pending empirical task. Finally, our attempt to fully cover the realm of maladaptive personality traits might have exceeded the possibilities of the PID-5 and the DAPP-BQ. One can think of a range of traits that are not manifestly collected by either instrument-dominance, ingenuity, need of attention, hyperactivity, self-esteem, cleanliness, machiavellianism, laziness, fanaticism, seductiveness-and whose potential impact on the final structure remains to be seen. Before we can embrace a dimensional taxonomy without reservations, we need to know whether all other instruments attain a similar degree of convergence.

Despite all the objections, we can conclude that the PID-5 and the DAPPBQ rest on a common underlying model of pathological personality traits, even if they measure partially different aspects of it. Many of their lower order facets are almost interchangeable using Supplement Figure S1 as guidance, whereas a few features are covered by only one of the instruments. As for the higher order domains, they do not exactly match with each other, but the PID-5 and the DAPP-BQ seem to measure complementary aspects of the same broad dimensions or to focus on distinct levels of a common hierarchical organization. These results have some implications for future revisions of the $D S M$ and ICD taxonomies. They provide unequivocal support for the idea that we have come across a universal structure of personality. In fact, they illustrate that most discrepancies between systems-for example, the presence or absence of compulsivity and subordination, the partition or not of dissociality into disinhibition and antagonism-are only apparent. They also suggest that a broader awareness of the hierarchical nature of personality, together with a willingness to move beyond the customary five dimensions, may be necessary to achieve full integration because many discrepancies between models are insolvable at the fifth level (see also F. Gutiérrez et al., 2014). Finally, they show that, even though a great deal of work remains to be done, we are closer than we have ever been to reaching an agreed empirically based diagnostic taxonomy, which is in itself encouraging. 


\section{REFERENCES}

American Psychiatric Association. (2013). Diagnostic and statistical manual of mental disorders (5th ed.). Washington, DC: Author.

Anderson, J. L., Sellbom, M., Bagby, R. M., Quilty, L. C., Veltri, C. O. C., Markon, K. E., \& Krueger, R. F. (2013). On the convergence between PSY-5 domains and PID-5 domains and facets: Implications for assessment of DSM-5 personality traits. Assessment, 20, 286-294.

Austin, E. J., \& Deary, I. J. (2000). The 'four As': A common framework for normal and abnormal personality? Personality and Individual Differences, 28, 977-995.

Bastiaens, T., Claes, L., Smits, D., De Clercq, B., De Fruyt, F., Rossi, G., . . . De Hert, M. (2016). The construct validity of the Dutch Personality Inventory for DSM-5 Personality Disorders (PID-5) in a clinical sample. Assessment, 23, 42-51.

Berghuis, H., Ingenhoven, T. J. M., van der Heijden, P. T., Rossi, G. M. P., \& Schotte, C. K. W. (2019). Assessment of pathological traits in DSM-5 personality disorders by the DAPP-BQ: How do these traits relate to the six personality disorder types of the alternative model? Journal of Personality Disorders, 33, 49-70.

Clark, L. A. (1993). The Schedule for Nonadaptive and Adaptive Personality (SNAP). Minneapolis, MN: University of Minnesota Press.

Clark, L. A., Livesley, W. J., Schroeder, M. L., \& Irish, S. L. (1996). Convergence of two systems for assessing specific traits of personality disorder. Psychological Assessment, 8, 294-303.

Clark, L. A., Shapiro, J. L., Daly, E., Vanderbleek, E. N., Negrón, M. R., \& Harrison, J. (2018). Empirically validated diagnostic and assessment methods. In W. J. Livesley \& R. Larstone (Eds.), Handbook of personality disorders. Theory, research, and treatment (2nd ed., pp. 341-366). New York, NY: Guilford Press.

Cloninger, C. R. (2000). A practical way to diagnosis personality disorder: A proposal. Journal of Personality Disorders, 14, 99-108.

Goldberg, L. R. (2006). Doing it all bass-ackwards: The development of hierarchical factor structures from the top down. Journal of Research in Personality, 40, 347-358.

Gutiérrez, F., Aluja, A., Peri, J. M., Calvo, N., Ferrer, M., Baillés, E., . . . Krueger, R. F. (2017). Psychometric properties of the Spanish PID5 in a clinical and a community sample. Assessment, 24, 326-336.

Gutiérrez, F., Vall, G., Peri, J. M., Gárriz, M., \& Garrido, J. M. (2014). A hierarchical model of normal and abnormal personality up to seven factors. Comprehensive Psychiatry, $55,326-335$.
Gutiérrez-Zotes, J. A., Gutiérrez, F., Valero, J., Gallego, E., Baillés, E., Torres, X., . . . Livesley, W. J. (2008). Structure of personality pathology in normal and clinical samples: Spanish validation of the DAPP-BQ. Journal of Personality Disorders, 22, 389-404.

Harkness, A. R., Finn, J. A., McNulty, J. L., \& Shields, S. M. (2012). The Personality Psychopathology-Five (PSY-5): Recent constructive replication and assessment literature review. Psychological Assessment, 24, 432-443.

Herpertz, S. C., Huprich, S. K., Bohus, M., Chanen, A., Goodman, M., Mehlum, L., ...Sharp, C. (2017). The challenge of transforming the diagnostic system of personality disorders. Journal of Personality Disorders, 31, 577-589.

Hooper, D., Coughlan, J., \& Mullen, M. (2008). Structural equation modelling: Guidelines for determining model fit. Electronic Journal of Business Research Methods, 6(1), 53-60.

Hopwood, C. J., Kotov, R., Krueger, R. F., Watson, D., Widiger, T. A., Althoff, R. R.,... Zimmermann, J. (2018). The time has come for dimensional personality disorder diagnosis. Personality and Mental Health, 12(1), 82-86.

Johnson, S. L., Leedom, L. J., \& Muhtadie, L. (2012). The dominance behavioral system and psychopathology: Evidence from selfreport, observational, and biological studies. Psychological Bulletin, 138, 692-743.

Krueger, R. F., Derringer, J., Markon, K. E., Watson, D., \& Skodol, A. E. (2012). Initial construction of a maladaptive personality trait model and inventory for DSM-5. Psychological Medicine, 42, 1879-1890.

Krueger, R. F., Eaton, N. R., Clark, L. A., Watson, D., Markon, K. E., Derringer, J., . . . Livesley, W. J. (2011). Deriving an empirical structure of personality pathology for DSM-5. Journal of Personality Disorders, 25, 170-191.

Kushner, S. C., Quilty, L. C., Tackett, J. L., \& Bagby, R. M. (2011). The hierarchical structure of the Dimensional Assessment of Personality Pathology (DAPP-BQ). Journal of Personality Disorders, 25, 504-516.

Livesley, W., \& Jackson, D. (2009). Manual for the Dimensional Assessment of Personality $\mathrm{Pa}$ thology-Basic Questionnaire. Port Huron, MI: Sigma Press.

Lorenzo-Seva, U. (2003). A factor simplicity index. Psychometrika, 68, 49-60.

Lorenzo-Seva, U., \& Ferrando, P. J. (2015, July). FACTOR. Unrestricted factor analysis. Release version 10.3.01 x64bits. Rovira i Virgili University, Tarragona, Spain. Retrieved from http://psico.fcep.urv.es/utilitats/factor 
Lorenzo-Seva, U., \& ten Berge, J. M. F. (2006). Tucker's congruence coefficient as a meaningful index of factor similarity. Methodology, 2(2), 57-64.

Markon, K. E., Krueger, R. F., \& Watson, D. (2005). Delineating the structure of normal and abnormal personality: An integrative hierarchical approach. Journal of Personality and Social Psychology, 88, 139-157.

Mestre-Pintó, J. I., Domingo-Salvany, A., MartínSantos, R., \& Torrens, M. (2014). Dual Diagnosis Screening Interview to identify psychiatric comorbidity in substance users: Development and validation of a brief instrument. European Addiction Research, 20, 41-48.

Morey, L. C., Krueger, R. F., \& Skodol, A. E. (2013). The hierarchical structure of clinician ratings of proposed DSM-5 pathological personality traits. Journal of Abnormal Psychology, 122, 836-841.

Mulder, R. T., \& Joyce, P. R. (1997). Temperament and the structure of personality disorder symptoms. Psychological Medicine, 27, 99-106.

Mulder, R. T., Newton-Howes, G., Crawford, M. J., \& Tyrer, P. J. (2011). The central domains of personality pathology in psychiatric patients. Journal of Personality Disorders, 25, 364-377.

Muñoz-Champel, A., Gutiérrez, F., Peri, J. M., \& Torrubia, R. (2018). Personality disorders are not as we thought: Hierarchical factor structure at the criterion level. Journal of Personality Assessment, 100, 16-29.

Ofrat, S., Krueger, R. F., \& Clark, L. A. (2018). Dimensional approaches to personality disorder classification. In W. J. Livesley \& R. Larstone (Eds.), Handbook of personality disorders. Theory, research, and treatment (2nd ed., pp. 72-87). New York, NY: Guilford Press.

Osborne, J. W. (2003). Effect sizes and the disattenuation of correlation and regression coefficients: Lessons from educational psychology. Practical Assessment, Research o Evaluation, 8(11), 1-7.

Osborne, J. W. (2014). Best practices in exploratory factor analysis. CreateSpace Independent Publishing Platform.
Reed, G. M. (2018). Progress in developing a classification of personality disorders for ICD11. World Psychiatry, 17, 227-229.

Rizopoulos, D. (2006). ltm: An R package for latent variable modelling and item response analysis. Journal of Statistical Software, 17, $1-25$.

Rushton, J. P., Irwing, P., \& Booth, T. (2010). A General Factor of Personality (GFP) in the personality disorders: Three studies of the Dimensional Assessment of Personality Pathology-Basic Questionnaire (DAPP-BQ). Twin Research and Human Genetics, 13, 301-311.

Tyrer, P., Crawford, M., Mulder, R., Blashfield, R., Farnam, A., Fossati, A., . . . Reed, G. M. (2011). The rationale for the reclassification of personality disorder in the 11th revision of the International Classification of Diseases (ICD-11). Personality and Mental Health, 5, 246-259.

Tyrer, P., Reed, G. M., \& Crawford, M. J. (2015). Classification, assessment, prevalence, and effect of personality disorder. Lancet, 385 , 717-726.

Van den Broeck, J., Bastiaansen, L., Rossi, G., Dierckx, E., De Clercq, B., \& Hofmans, J. (2014). Hierarchical structure of maladaptive personality traits in older adults: Joint factor analysis of the PID-5 and the DAPPBQ. Journal of Personality Disorders, 28, 198-211.

Watson, D., Stasik, S. M., Ro, E., \& Clark, L. A. (2013). Integrating normal and pathological personality: Relating the DSM-5 traitdimensional model to general traits of personality. Assessment, 20, 312-326.

Widiger, T. A. (2018). Official classification systems. In W. J. Livesley \& R. Larstone (Eds.), Handbook of personality disorders. Theory, research, and treatment (2nd ed., pp. 4771). New York, NY: Guilford Press.

Widiger, T. A., \& Simonsen, E. (2005). Alternative dimensional models of personality disorder: Finding a common ground. Journal of Personality Disorders, 19, 110-130.

Williams, M. N., Gómez-Grajales, C. A., \& Kurkiewicz, D. (2013). Assumptions of multiple regression: Correcting two misconceptions. Practical Assessment, Research \& Evaluation, 18(11), 1-14. 
SUPPLEMENTAL MATERIAL: Towards an Integrated Model of Pathological Personality Traits: Common Hierarchical Structure of the PID-5 and the DAPP-BQ

Supplemental Table S1. Means (SD) Expressed in T Scores and Cronbach's $\alpha$ Coefficients for the PID-5 and the DAPP-BQ.

\begin{tabular}{|c|c|c|}
\hline & $M(S D)$ & $\alpha$ \\
\hline \multicolumn{3}{|l|}{ PID-5 Traits } \\
\hline Depressivity & $69.0(17.9)$ & .94 \\
\hline Anhedonia & $65.5(15.7)$ & .86 \\
\hline Suspiciousness & $60.8(15.6)$ & .85 \\
\hline Emotional Lability & $57.5(12.7)$ & .89 \\
\hline Perceptual Dysregulation & $58.9(15.6)$ & .87 \\
\hline Anxiousness & $61.5(12.5)$ & .89 \\
\hline Rigid Perfectionism & $53.6(12.9)$ & .91 \\
\hline Risk Taking & $51.8(13.8)$ & .90 \\
\hline Withdrawal & $58.7(15.3)$ & .92 \\
\hline Hostility & $58.6(13.4)$ & .88 \\
\hline Attention Seeking & $53.2(12.5)$ & .93 \\
\hline Distractibility & $61.0(13.8)$ & .92 \\
\hline Separation Insecurity & $53.6(13.6)$ & .91 \\
\hline Callousness & $55.7(14.6)$ & .86 \\
\hline Irresponsibility & $60.6(15.2)$ & .81 \\
\hline Impulsivity & $58.1(13.5)$ & .94 \\
\hline Submissiveness & $54.9(13.0)$ & .90 \\
\hline Unusual Beliefs \& Exper. & $53.9(14.2)$ & .85 \\
\hline Perseveration & $59.7(12.9)$ & .81 \\
\hline Eccentricity & $60.3(14.1)$ & .95 \\
\hline Restricted Affectivity & $53.8(12.7)$ & .83 \\
\hline Intimacy Avoidance & $54.0(13.1)$ & .88 \\
\hline Manipulativeness & $55.7(13.7)$ & .83 \\
\hline Deceitfulness & $57.3(14.4)$ & .88 \\
\hline Grandiosity & $50.2(11.5)$ & .82 \\
\hline \multicolumn{3}{|l|}{ DAPP-BQ Traits } \\
\hline Identity Problems & $66.0(12.2)$ & .93 \\
\hline Affective Lability & $61.5(13.4)$ & .92 \\
\hline Cognitive Distortion & $56.8(14.5)$ & .91 \\
\hline Anxiousness & $61.2(12.9)$ & .93 \\
\hline Stimulus Seeking & $54.0(13.1)$ & .88 \\
\hline Suspiciousness & $56.3(14.5)$ & .91 \\
\hline Narcissism & $55.8(12.4)$ & .90 \\
\hline Insecure Attachment & $51.0(13.4)$ & .94 \\
\hline Callousness & $53.6(12.1)$ & .83 \\
\hline Compulsivity & $48.7(12.9)$ & .91 \\
\hline Oppositionality & $59.6(13.0)$ & .88 \\
\hline Submissiveness & $57.1(13.3)$ & .88 \\
\hline Social Avoidance & $59.6(13.8)$ & .90 \\
\hline Intimacy Problems & $53.8(13.3)$ & .84 \\
\hline Restricted Expression & $53.8(13.0)$ & .87 \\
\hline Rejection & $54.0(12.4)$ & .86 \\
\hline Conduct Problems & $56.5(13.5)$ & .85 \\
\hline Self-Harm & $68.5(25.6)$ & .94 \\
\hline
\end{tabular}


Supplemental Table S2. Promax-Rotated One- to Six-Factor Solutions for the PID-5 and the DAPP-BQ.

\begin{tabular}{|c|c|c|c|c|c|c|c|c|c|c|}
\hline & \multirow[b]{2}{*}{ 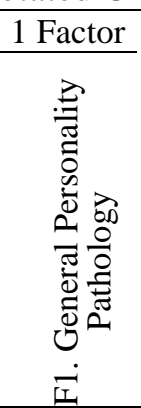 } & \multicolumn{2}{|c|}{2 Factors } & \multicolumn{3}{|c|}{3 Factors } & \multicolumn{4}{|c|}{4 Factors } \\
\hline & & 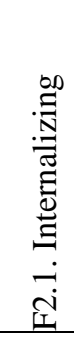 & 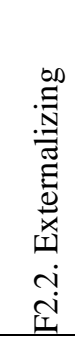 & 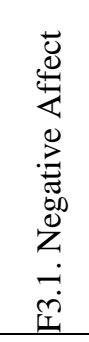 & 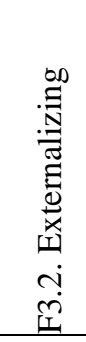 & 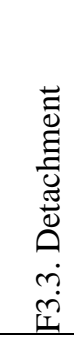 & 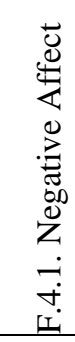 & 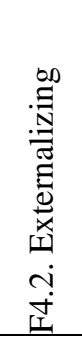 & 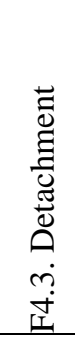 & 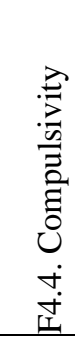 \\
\hline DAPP Anxiousness & .78 & .88 & -.07 & .98 & -.17 & -.04 & .98 & -.17 & -.05 & .07 \\
\hline PID Anxiousness & .71 & .78 & -.04 & .92 & -.14 & -.11 &.$\overline{91}$ & -.14 & -.10 & .14 \\
\hline DAPP Identity Problems &.$\overline{79}$ & .94 & -.12 & .78 & -.13 & .31 & .80 & -.12 & .29 & -.02 \\
\hline PID Depressivity &.$\overline{79}$ & .93 & -.10 & .80 & -.12 & .27 & .83 & -.12 & .23 & -.05 \\
\hline PID Anhedonia & .61 & .85 & -.24 & .58 & -.21 & .46 & .62 & -.20 & .42 & -.07 \\
\hline DAPP Insecure Attachment &.$\overline{59}$ &.$\overline{45}$ & .21 & .80 & .06 & $\underline{-. \overline{42}}$ &.$\overline{78}$ & .05 &.$- \overline{41}$ & .09 \\
\hline DAPP Submissiveness &.$\overline{59}$ & .76 & -.16 & .85 & -.25 & -.07 & .86 & -.26 & -.09 & -.01 \\
\hline PID Separation Insecurity &.$\overline{53}$ &.$\overline{42}$ & .17 &.$\overline{74}$ & .03 & -.39 &.$\overline{.73}$ & .02 & -.39 & .04 \\
\hline DAPP Affective Lability &.$\overline{79}$ &.$\overline{56}$ & .34 &.$\overline{73}$ & .23 & -.13 &.$\overline{72}$ & .23 & -.13 & .06 \\
\hline DAPP Social Avoidance & .67 &.$\overline{86}$ & -.18 & .67 & -.18 & .34 & .69 & -.16 & .34 & .10 \\
\hline PID Emotional Lability & .69 &.$\overline{53}$ & .24 &.$\overline{76}$ & .13 & -.23 &.$\overline{74}$ & .12 & -.23 & .04 \\
\hline PID Perseveration & .73 & .67 & .12 & .68 & .07 & .07 & .68 & .07 & .07 & .09 \\
\hline DAPP Oppositionality & .65 & .57 & .14 & .55 & .10 & .11 & .62 & .06 & .02 & -.42 \\
\hline PID Distractibility & .65 & .59 & .11 & .56 & .08 & .13 & .61 & .05 & .05 & -.37 \\
\hline PID Submissiveness &.$\overline{42}$ &.$\overline{58}$ & -.17 &.$\overline{68}$ & -.24 & -.10 &.$\overline{68}$ & -.24 & -.10 & .07 \\
\hline PID Suspiciousness & .72 & .56 & .24 & .53 & .21 & .15 & .52 & .23 & .17 & .19 \\
\hline DAPP Suspiciousness & .75 &.$\overline{53}$ & .32 &.$\overline{.52}$ & .28 & .13 &.$\overline{50}$ & .30 & .16 & .21 \\
\hline DAPP Self-Harm & $\underline{.56}$ & $\underline{.58}$ & .02 & .53 & -.01 & .14 &.$\overline{54}$ & -.01 & .12 & -.01 \\
\hline PID Manipulativeness & $\overline{.33}$ &.$- \overline{34}$ & .84 & $-\overline{25}$ & .82 & -.03 &.$- \overline{28}$ & .84 & -.01 & .05 \\
\hline PID Deceitfulness & .51 & -.04 &.$\overline{72}$ & .00 & .69 & .05 & -.02 & .70 & .05 & .00 \\
\hline DAPP Callousness &.$\overline{55}$ & .02 &.$\overline{70}$ & -.10 & .74 & .28 & -.12 & .76 & .29 & .08 \\
\hline PID Attention Seeking & .47 & -.12 & .77 & .18 &. .66 & -.33 & .14 & .67 & -.31 & .09 \\
\hline DAPP Rejection &.$\overline{45}$ & -.17 & .81 & -.03 & .76 & -.09 & -.09 &.$\overline{79}$ & -.04 & .23 \\
\hline DAPP Narcissism & .61 & .18 & .57 & .49 & .45 & -.34 & .44 & .46 & -.30 & .23 \\
\hline PID Grandiosity & .29 & -.20 & .62 & -.12 & .59 & -.04 & -.19 & .65 & .03 & .37 \\
\hline PID Callousness & .49 & .03 & .60 & -.20 & .69 & .42 & -.20 & .71 & .41 & -.02 \\
\hline DAPP Intimacy Problems & $\overline{.21}$ & .39 & $-\overline{20}$ & -.13 & $-\overline{08}$ &.$\overline{82}$ & -.09 &.$- \overline{05}$ &.$\overline{79}$ & -.04 \\
\hline PID Intimacy Avoidance & .19 & .28 & -.10 & -.20 & .03 & .74 & -.16 & .05 & .72 & -.06 \\
\hline DAPP Restricted Expression & .37 & .57 & -.21 & .14 & -.12 & .69 & .16 & -.09 & .69 & .10 \\
\hline PID Withdrawal & .43 &.$\overline{59}$ & -.17 & .12 & -.07 &.$\overline{78}$ & .16 & -.04 & .77 & .03 \\
\hline PID Restricted Affectivity & $\overline{.29}$ &.$\overline{24}$ & .08 & -.18 & .20 & .66 & -.16 & .23 & .66 & .05 \\
\hline DAPP Stimulus Seeking & .47 & -.10 & .74 & .09 & .67 & -.16 & .09 & .66 & -.23 & -.35 \\
\hline PID Risk Taking &.$\overline{22}$ & -.31 &.$\overline{66}$ & -.20 &.$\overline{63}$ & -.08 & -.20 &.$\overline{62}$ & -.13 & -.27 \\
\hline PID Impulsivity & .54 & .12 & .54 & .25 & .48 & -.08 & .27 &.$\overline{46}$ & -.16 & -.34 \\
\hline PID Irresponsibility & .62 & .34 & .38 & .26 & .37 & .22 & .30 &.$\overline{36}$ & .13 & $\underline{-.48}$ \\
\hline DAPP Conduct Problems & .55 & .11 & .58 & .06 & .58 & .18 & .07 & .58 & .14 & -.18 \\
\hline PID Unusual Beliefs \& Experiences &.$\overline{59}$ & .30 &.$\overline{40}$ & .32 & .36 & .06 & .30 & .38 & .08 & .11 \\
\hline PID Perceptual Dysregulation &.$\overline{76}$ & .58 &.$\overline{27}$ & .56 & .23 & .14 & .56 & .24 & .12 & .01 \\
\hline DAPP Cognitive Distortion & .82 &.$\overline{73}$ & .16 & .69 & .11 & .16 &.$\overline{.71}$ & .11 & .13 & -.03 \\
\hline PID Eccentricity & .71 & .54 & .25 &.$\overline{40}$ & .25 & .31 &.$\overline{41}$ & .27 & .30 & .04 \\
\hline PID Rigid Perfectionism & .38 &.$\overline{29}$ & .14 &.$\overline{37}$ & .09 & -.06 &.$\overline{31}$ & .14 & .05 & .67 \\
\hline DAPP Compulsivity & .15 & .13 & .03 & .23 & -.01 & -.12 & .17 & .04 & .00 & .78 \\
\hline PID Hostility & $\underline{.66}$ & .22 & .59 & .19 & $\underline{.57}$ & .16 & .16 & .18 &. .59 &.$\overline{16}$ \\
\hline $\begin{array}{l}\text { Congruence with van den Broeck et } \\
\text { al. (2014) }\end{array}$ & .98 & .90 & .81 & .87 & .82 & .70 & .88 & .82 & .70 & .51 \\
\hline
\end{tabular}

Note. Factor loadings $\geq .30$ are shown in bold type; the highest loading for each facet is underlined. 
Supplemental Table S2 (Cont.). Promax-Rotated One- to Six-Factor Solutions for the PID-5 and the DAPP-BQ. 5 Factors

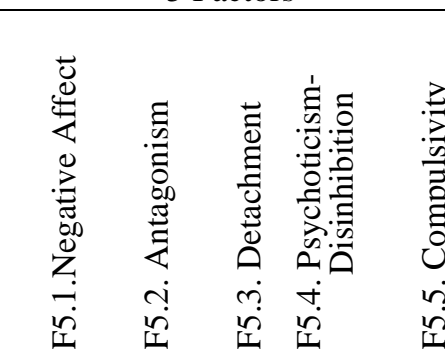

\begin{tabular}{l}
\hline DAPP Anxiousness \\
PID Anxiousness \\
DAPP Identity Problems \\
PID Depressivity \\
PID Anhedonia \\
DAPP Insecure Attachment \\
DAPP Submissiveness \\
PID Separation Insecurity
\end{tabular}

DAPP Affective Lability

DAPP Social Avoidance

PID Emotional Lability

PID Perseveration

DAPP Oppositionality

PID Distractibility

PID Submissiveness

PID Suspiciousness

DAPP Suspiciousness

DAPP Self-Harm

PID Manipulativeness

PID Deceitfulness

DAPP Callousness

PID Attention Seeking

DAPP Rejection

DAPP Narcissism

PID Grandiosity

PID Callousness

DAPP Intimacy Problems

PID Intimacy Avoidance

DAPP Restricted Expression

PID Withdrawal

PID Restricted Affectivity

DAPP Stimulus Seeking

PID Risk Taking

PID Impulsivity

PID Irresponsibility

DAPP Conduct Problems

PID Unusual Beliefs \& Experiences

PID Perceptual Dysregulation

DAPP Cognitive Distortion

PID Eccentricity

PID Rigid Perfectionism

DAPP Compulsivity

PID Hostility

Congruence with van den Broeck et al. (2014)

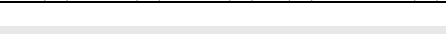

6 Factors

\begin{tabular}{|c|c|c|c|c|c|c|c|c|c|c|}
\hline .95 & -.08 & -.05 & -.03 & .04 & .99 & -.10 & -.05 & -.07 & .04 & .09 \\
\hline$\overline{.88}$ & -.05 & -.10 & -.05 & .12 &.$\overline{99}$ & -.10 & -.11 & -.15 & .12 & -.02 \\
\hline .81 & .00 & .30 & -.11 & -.07 & .90 & -.04 & .28 & -.19 & -.07 & -.02 \\
\hline .78 & -.08 & .24 & .02 & -.06 & .82 & -.10 & .23 & .00 & -.07 & .06 \\
\hline .69 & .01 & .45 & -.26 & -.15 & .73 & -.02 & .43 & -.29 & -.14 & .03 \\
\hline$\overline{.77}$ & .11 & -.41 & .01 & .06 &.$\overline{79}$ & .09 & -.40 & -.03 & .06 & .10 \\
\hline .88 & -.11 & -.09 & -13 & -.05 & .67 & -.05 & -.05 & .09 & -.05 &. .54 \\
\hline .77 & .13 & -.40 & -.09 & -.02 & .75 & .12 & -.38 & -.11 & -.02 & .15 \\
\hline$\overline{.56}$ & .02 & -.14 & .39 & .13 &.$\overline{79}$ & -.05 & -.18 & .17 & .12 & -.29 \\
\hline$\overline{.70}$ & .00 & .36 & -.19 & .05 & .65 & .00 & .35 & -.09 & .05 & .18 \\
\hline. & -.09 & -.24 & .40 & . 12 & .72 & -.13 & -.26 & .25 & .11 & -.14 \\
\hline .62 & .06 & .08 & .08 & .09 & $\underline{.63}$ & .06 & .08 & .09 & .09 & .09 \\
\hline .62 & .03 & .02 & .16 & -.43 &.$\overline{58}$ & .05 & .03 & .10 & -.44 & .14 \\
\hline .57 & -.04 & .05 & .24 & -.34 & .54 & -.03 & .06 & .20 & -.35 & .12 \\
\hline .72 & -.05 & -.09 & -.22 & .01 & .50 & .02 & -.06 & .02 & .02 & .54 \\
\hline .44 & .18 & .18 & .11 & .19 & .51 & .16 & .17 & .08 & .18 & -.04 \\
\hline 42 & .23 & .16 & .14 & .22 &.$\overline{49}$ & .22 & .15 & .11 & .21 & -.05 \\
\hline 38 & -.20 & .12 & .34 & .08 & .42 & -.19 & .12 & .32 & .07 & .00 \\
\hline 19 & .83 & -.01 & .01 & -.04 & -.22 & .87 & .00 & -.02 & -.04 & .01 \\
\hline .08 & .75 & .06 & -.04 & -.09 & -.02 & .81 & .08 & .00 & -.09 & .17 \\
\hline .05 & .77 & .31 & -.01 & .00 & -.02 & .78 & .30 & -.06 & .00 & -.08 \\
\hline 19 & .65 & -.32 & .06 & .03 & .11 & .70 & -.29 & .08 & .03 & .17 \\
\hline .08 & .70 & -.04 & .11 & 19 & .05 & .69 & -.05 & -.04 & .19 & -.24 \\
\hline .52 & .57 & -.31 & -.11 & .14 & .43 & .61 & -.28 & -.05 & .15 & .24 \\
\hline .20 & .58 & .03 & .06 & .34 & -.23 & .61 & .04 & .14 & .35 & .02 \\
\hline 19 &.$\overline{59}$ & .42 & .15 & -.05 & -.11 & .59 & .40 & .05 & -.05 & -.18 \\
\hline .13 &.$- \overline{08}$ & .81 & .01 & -.02 & -.07 &.$- \overline{09}$ & .78 & .02 & -.03 & -.11 \\
\hline .22 & -.03 &.$\overline{73}$ & .09 & -.03 & -.18 & -.02 &.$\overline{71}$ & .11 & -.03 & -.10 \\
\hline .18 & .03 &.$\overline{71}$ & -.19 & .06 & .12 & .05 &.$\overline{.71}$ & -.05 & .07 & .12 \\
\hline .10 & -.04 & .78 & -.01 & .04 & .14 & -.05 &.$\overline{76}$ & .04 & .04 & -.05 \\
\hline .12 & .30 & .68 & -13 & .00 & -.16 & .32 & .68 & -.04 & .01 & .05 \\
\hline .09 & .20 & -.25 & .77 & -.23 & -.01 & .24 &.$- \overline{25}$ & .57 & -.25 & -.13 \\
\hline .38 & .18 & -.14 & .70 & -.14 & -.31 & .22 & -.14 & .55 & -.17 & -.15 \\
\hline .14 & .12 & -.17 &.$\overline{59}$ & -.25 & .24 & .12 & -.18 &.$\overline{38}$ & -.27 & -.16 \\
\hline .29 & .20 & .13 & .31 & -.47 & .25 & .24 & .14 &.$\overline{24}$ & -.48 & .09 \\
\hline .05 & .28 & .14 & .49 & -.11 & .05 & .29 & .12 & .34 & -.13 & -.17 \\
\hline .02 & -.05 & .07 & .67 & .28 & -.05 & .00 & .08 & .83 & .28 & .11 \\
\hline .32 & -.10 & .12 & .58 & .14 & .24 & -.06 & .14 &.$\overline{72}$ & .14 & .19 \\
\hline 48 & -.18 & .13 & .52 & .09 & .43 & -.15 & .14 & .61 & .08 & .17 \\
\hline .23 & .02 & .31 & .42 & .14 & .22 & .05 & .31 &.$\overline{48}$ & .13 & .06 \\
\hline .22 & .15 & .04 & -.04 & .68 & .27 & .14 & .04 & .05 &. .68 & -.02 \\
\hline .06 & .05 & .00 & -.07 & .82 & .08 & .05 & .00 & .09 &.$\overline{82}$ & .02 \\
\hline .12 & .41 & .16 & .28 & .05 & .36 & .37 & .13 & .01 & .04 & $\underline{-.40}$ \\
\hline 8 & .75 & .73 & .78 & .62 & -- & -- & -- & -- & -- & - \\
\hline
\end{tabular}

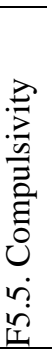


Supplemental Table S3. Between- and Within-level Pearson's Correlations for the Seven Levels of the Promax-Rotated Personality Hierarchy.

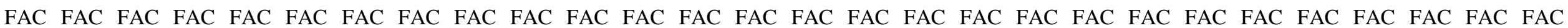

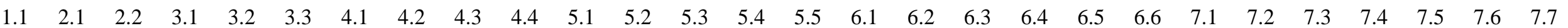

FAC 1.1

FAC 2.1

FAC 2.2

FAC 3.1

FAC 3.2

FAC 3.3

FAC 4.1

FAC 4.2

FAC 4.3

FAC 4.4

FAC 5.1

FAC 5.2

FAC 5.3

FAC 5.4

FAC 5.5

FAC 6.1

FAC 6.2

FAC 6.3

FAC 6.4

FAC 6.5

FAC 6.6

FAC 7.1

FAC 7.2

$.95^{* *}-$

$.78^{* *} .55^{* *}$

$.95^{* *} .97^{* *} \quad .60^{* *}-$

$.72^{* *} .47^{* *} .99^{* *} \quad .50^{* *}$

$.49^{* *} \quad .57^{* *} \cdot .20^{* *} \cdot .37^{* *} .24^{* *}-$

$.95^{* *} .98^{* *} .60^{* *} 1.00^{* *} \quad .50^{* *} \quad .39^{* *}-$

$.73^{* *} .49^{* *} .99^{* *} .52^{* *} \mathbf{1 . 0 0}^{* *} .24^{* *} .52^{* *}-$

$\begin{array}{ccccccccc}.43^{* *} & .51^{* *} & .13^{* *} & .31^{* *} & .18^{* *} & .99^{* *} & .32^{* *} & .18^{* *} & - \\ -.05 & -.05 & -.05 & .00 & -.07 & -.17^{* *} & -.05 & -.07 & -.10^{*}\end{array}$

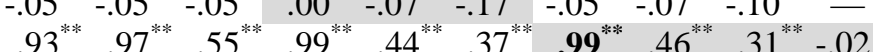

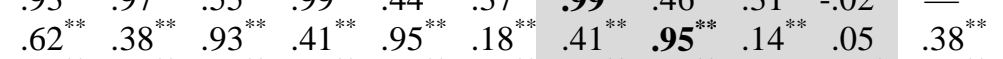

$.48^{* *} .56^{* *} .18^{* *} .36^{* *} .22^{* *} 1.00^{* *} .38^{* *} .22^{* *} \mathbf{1 . 0 0}^{* *}-.11^{*} .36^{* *} \overline{16}^{* *}$

$81^{* *} .65^{* *} .84^{* *} \quad 67^{* *} \quad 81^{* *} 33^{* *} \quad \mathbf{6 8}^{* *} \quad \mathbf{8 2}^{* *} \quad 26^{* *}-27^{* *} \quad 60^{* *} \quad .60^{* *} \quad 1^{* *}$

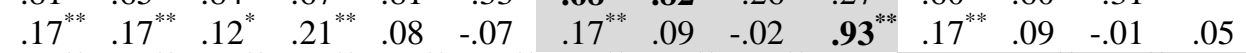

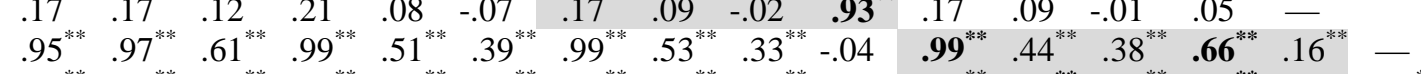

$.65^{* *} .40^{* *} .94^{* *} .44^{* *} \quad .96^{* *} \quad .18^{* *} .44^{* *} \quad .96^{* *} \quad .14^{* *} .03 \quad .40^{* *} \mathbf{1 . 0 0}^{* *} \quad .17^{* *} .64^{* *} .09 \quad .46^{* *}-$

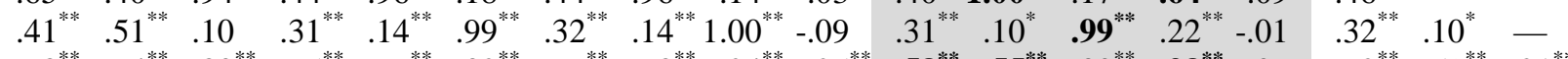

$\begin{array}{llllllllllllllllllll}.78^{* *} & .64^{* *} & .80^{* *} & .65^{* *} & .77^{* *} & .32^{* *} & .66^{* *} & .78^{* *} & .24^{* *} & -.25^{* *} & .58^{* *} & .55^{* *} & .30^{* *} & .98^{* *} & .07 & .62^{* *} & .59^{* *} & .21^{* *} & -\end{array}$

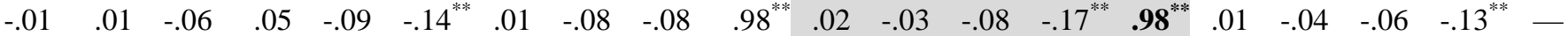

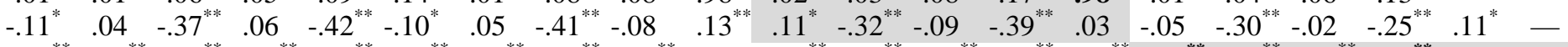

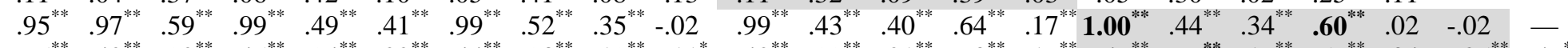

FAC 7.3

FAC 7.4

FAC 7.5

$\begin{array}{lllllllllllllllllllllll}.65^{* *} & .42^{* *} & .92^{* *} & .45^{* *} & .94^{* *} & .22^{* *} & .44^{* *} & .93^{* *} & .19^{* *} & .11^{*} & .42^{* *} & .99^{* *} & .21^{* *} & .60^{* *} & .16^{* *} & .46^{* *} & .99^{* *} & .16^{* * *} & .56^{* *} & .04 & -.25^{* *} & .46^{* *} & -\end{array}$

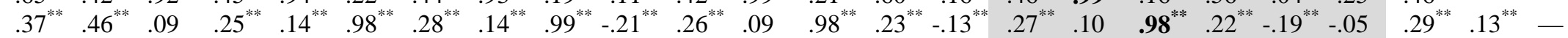

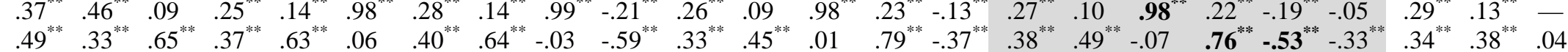

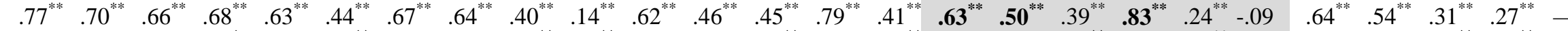

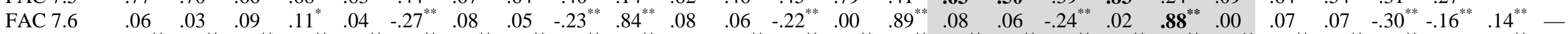

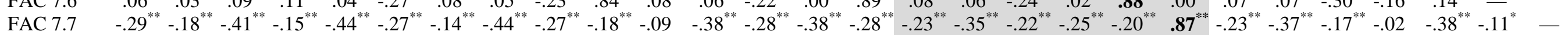

** $\mathrm{p}<.01 * \mathrm{p}<.05$. Between-level correlations are shaded in grey and are in bold type if $\mathrm{r} \geq .50$ 
Supplemental Table S4. Varimax-Rotated One- to Seven-Factor Solutions for the PID-5 and the DAPP-BQ.

\begin{tabular}{|c|c|c|c|c|c|c|c|c|c|c|}
\hline & \multirow[b]{2}{*}{ 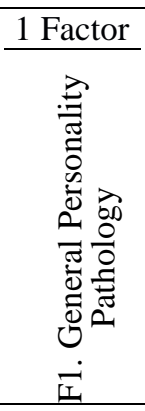 } & \multicolumn{2}{|c|}{2 Factors } & \multicolumn{3}{|c|}{3 Factors } & \multicolumn{4}{|c|}{4 Factors } \\
\hline & & 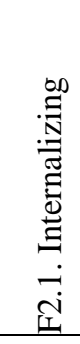 & 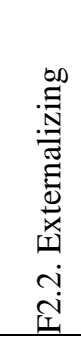 & 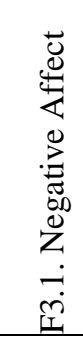 & 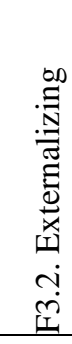 & 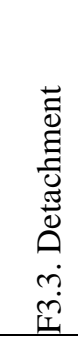 & 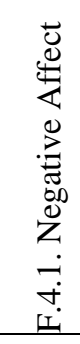 & 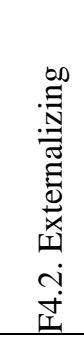 & 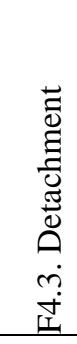 & 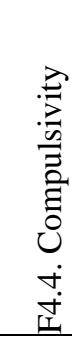 \\
\hline DAPP Anxiousness & .78 & .82 & .21 & .89 & .06 & .09 & .89 & .07 & .07 & .04 \\
\hline PID Anxiousness & $\underline{.72}$ &.$\overline{.74}$ & .21 &.$\overline{84}$ & .06 & .02 &.$\overline{83}$ & .07 & .00 & .12 \\
\hline DAPP Identity Problems & .79 & .87 & .16 & .77 & .09 & .41 & .79 & .09 & .38 & -.05 \\
\hline PID Depressivity & .80 & .86 & .18 & .79 & .09 & .37 & .81 & .09 & .34 & -.08 \\
\hline PID Anhedonia & .63 & .78 & .00 & .60 & -.03 & .53 & .62 & -.03 & .51 & -.09 \\
\hline DAPP Insecure Attachment & .61 &. .48 & .38 & .72 & .21 & -.30 & .71 & .21 & -.32 & .08 \\
\hline DAPP Submissiveness & $\underline{.60}$ & $\underline{.69}$ & .07 & .77 & -.06 & .03 & .77 & -.06 & .00 & -.02 \\
\hline PID Separation Insecurity &. .55 &.$\underline{.44}$ & .32 & .67 & .17 & -.29 & $\underline{.66}$ & .16 & -.32 & .02 \\
\hline DAPP Affective Lability &.$\overline{.79}$ & $\underline{.61}$ & .51 & .74 & .38 & .00 & .74 & .39 & -.03 & .03 \\
\hline DAPP Social Avoidance &.$\overline{.68}$ & $\underline{.80}$ & .07 & $\underline{.68}$ & .01 & .43 &.$\overline{.68}$ & .02 & .42 & .09 \\
\hline PID Emotional Lability & .70 &.$\overline{.57}$ & .42 &.$\overline{.73}$ & .28 & -.11 &.$\overline{.73}$ & .28 & -.14 & .01 \\
\hline PID Perseveration &.$\overline{.74}$ & .68 & .32 &.$\overline{.70}$ & .23 & .18 &.$\overline{70}$ & .24 & .16 & .07 \\
\hline DAPP Oppositionality & .66 & .60 & .31 & .59 & .24 & .21 & .62 & .21 & .13 & -.47 \\
\hline PID Distractibility & .67 & $\underline{.61}$ & .29 & .60 & .22 & .23 &.$\overline{.63}$ & .20 & .16 & -.42 \\
\hline PID Submissiveness &.$\overline{43}$ &.$\overline{.54}$ & .00 &.$\overline{.61}$ & -.11 & -.03 &.$\underline{.61}$ & -.11 & -.04 & .08 \\
\hline PID Suspiciousness & .73 &.$\underline{.61}$ & .40 & .60 & .34 & .25 & .60 & .36 & .25 & .17 \\
\hline DAPP Suspiciousness & .76 & .60 & .47 & .60 & .41 & .23 & .59 & .42 & .23 & .19 \\
\hline DAPP Self-Harm &.$\overline{58}$ &.$\overline{.58}$ & .18 & .56 & .12 & .23 & .56 & .12 & .20 & -.04 \\
\hline PID Manipulativeness & .34 & -.12 & .73 & -.05 & .76 & .00 & -.06 & .77 & .01 & .02 \\
\hline PID Deceitfulness &.$\overline{.53}$ & .14 &.$\overline{.70}$ & .18 & .70 & .11 & .18 & .70 & .09 & -.03 \\
\hline DAPP Callousness & .57 & .21 & .68 & .14 & .73 & .33 & .14 & .74 & .33 & .05 \\
\hline PID Attention Seeking &.$\overline{.49}$ & .06 & .74 & .28 & .67 & -.26 & .26 &.$\underline{.68}$ & -.27 & .08 \\
\hline DAPP Rejection & .47 & .03 & .75 & .13 & .74 & -.03 & .11 & .76 & -.01 & .22 \\
\hline DAPP Narcissism & .63 & .31 & .64 & .53 & .52 & -.24 & .51 & .54 & -.24 & .22 \\
\hline PID Grandiosity & .30 & -.05 & .58 & .01 & .59 & -.02 & -.01 & .62 & .03 & .40 \\
\hline PID Callousness & .50 & .19 & .59 & .05 & .67 & .45 & .06 &. .67 & .44 & -.06 \\
\hline DAPP Intimacy Problems & .22 & .38 & -.15 & .00 & -.03 & $\underline{.81}$ & .03 & -.02 & $\underline{.80}$ & -.06 \\
\hline PID Intimacy Avoidance & .20 & .29 & -.07 & -.06 & .05 & .75 & -.03 & .06 & .74 & -.08 \\
\hline DAPP Restricted Expression & .38 & .54 & -.10 & .23 & -.03 &.$\overline{.72}$ & .25 & -.01 & .73 & .09 \\
\hline PID Withdrawal & .45 & .58 & -.04 & .25 & .03 & .79 & .27 & .04 & .79 & .01 \\
\hline PID Restricted Affectivity & .30 & .29 & .11 & -.01 & .22 & .68 & .00 & .24 & .69 & .04 \\
\hline DAPP Stimulus Seeking & .49 & .08 & .72 & .21 &.$\underline{.69}$ & -.10 & .23 & .66 & -.16 & -.41 \\
\hline PID Risk Taking & .23 & -.15 & .59 & -.08 & .61 & -.06 & -.06 & .59 & -.10 & -.33 \\
\hline PID Impulsivity &. .55 & .25 & .59 & .34 & .54 & -.01 & .37 & .52 & -.08 & -.41 \\
\hline PID Irresponsibility &.$\underline{.63}$ & .44 & .47 & .38 & .46 & .31 & .42 & .43 & .22 & $\underline{-.53}$ \\
\hline DAPP Conduct Problems &.$\underline{.57}$ & .26 & $\underline{.61}$ & .22 & $\underline{.62}$ & .25 & .24 & $\underline{.61}$ & .20 & $\overline{-.24}$ \\
\hline PID Unusual Beliefs \& Experiences & .61 & .39 &. .49 & .42 & .45 & .14 & .41 & .46 & .14 & .10 \\
\hline PID Perceptual Dysregulation & .77 &.$\underline{.63}$ & .44 & .63 & .37 & .24 & .64 & .37 & .21 & -.03 \\
\hline DAPP Cognitive Distortion &.$\underline{.82}$ & .74 & .37 &.$\overline{.73}$ & .29 & .27 &.$\overline{.74}$ & .29 & .24 & -.07 \\
\hline PID Eccentricity & .72 &.$\underline{.61}$ & .40 &. .52 & .37 & .40 & .52 & .38 & .38 & .01 \\
\hline PID Rigid Perfectionism & .39 & .32 & .23 & .39 & .16 & -.02 & $\overline{.35}$ & .21 & .05 & .70 \\
\hline DAPP Compulsivity & .16 & .14 & .08 & .23 & .02 & -.12 & .17 & .08 & -.02 & .82 \\
\hline PID Hostility & .68 & .37 & .64 & .36 & .63 & .24 & .36 & .63 & .22 & .00 \\
\hline Congruence with promax rotation & 1.00 & .98 & .94 & .98 & .95 & .97 & .97 & .95 & .98 & .99 \\
\hline Congruence with van den Broeck et & .98 & .94 & .94 & .91 & .94 & .84 & .92 & .94 & .82 & .47 \\
\hline
\end{tabular}


Supplemental Table S4 (Cont.). Varimax-Rotated One- to Seven-Factor Solutions for the PID-5 and the DAPP-BQ. 5 Factors 6 Factors

\begin{tabular}{|c|c|c|c|c|c|c|c|c|c|c|c|}
\hline & & & & & & & & & & & \\
\hline & 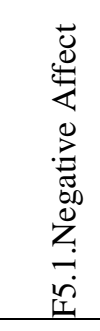 & 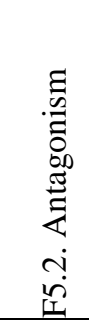 & 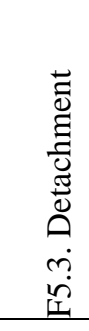 & 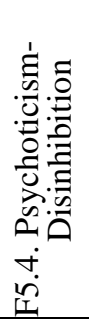 & 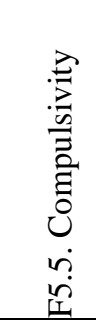 & 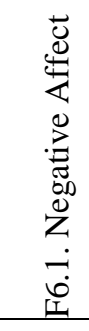 & 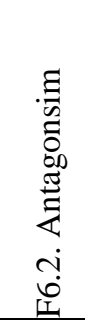 & 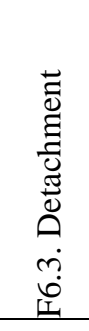 & 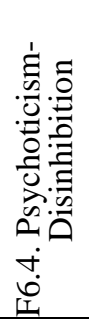 & 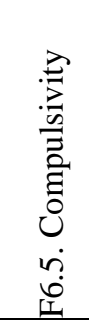 & 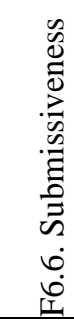 \\
\hline DAPP Anxiousness & .87 & .06 & .08 & .17 & .08 & .88 & .05 & .07 & .10 & .05 & .14 \\
\hline PID Anxiousness & .82 & .07 & .02 & .13 & .15 & .86 & .06 & .00 & .03 & .12 & .01 \\
\hline DAPP Identity Problems &.$\overline{78}$ & .10 & .40 & .13 & -.04 &.$\overline{82}$ & .09 & .38 & .03 & -.07 & .02 \\
\hline PID Depressivity & .78 & .06 & .35 & .22 & -.04 & .79 & .05 & .34 & .15 & -.07 & .10 \\
\hline PID Anhedonia & .64 & .05 & .52 & -.03 & -.13 &.$\overline{66}$ & .03 & .50 & -.11 & -.16 & .05 \\
\hline DAPP Insecure Attachment & .71 & .21 & -.31 & .15 & .10 & .72 & .20 & -.32 & .09 & .07 & .10 \\
\hline DAPP Submissiveness &.$\overline{79}$ & -.01 & .02 & .01 & -.04 & .64 & -.01 & .04 & .09 & -.03 & .59 \\
\hline PID Separation Insecurity & .68 & .21 & -.31 & .06 & .00 & .67 & .20 & -.31 & .00 & -.02 & .16 \\
\hline DAPP Affective Lability & .65 & .22 & -.02 & .48 & .16 & .77 & .22 & -.04 & .34 & .11 & -.21 \\
\hline DAPP Social Avoidance & .69 & .07 & .43 & .01 & .07 & .66 & .06 & .43 & -.01 & .05 & .22 \\
\hline PID Emotional Lability & .64 & .11 & -.12 & .46 & .15 & .72 & .11 & -.14 & .36 & .11 & -.09 \\
\hline PID Perseveration & .67 & .20 & .18 & .23 & .12 & .67 & .19 & .17 & .19 & .09 & .14 \\
\hline DAPP Oppositionality & .61 & .16 & .14 & .28 & -.44 & .59 & .16 & .14 & .25 & -.46 & .17 \\
\hline PID Distractibility &.$\overline{60}$ & .11 & .17 & .33 & -.36 &.$\overline{58}$ & .12 & .17 & .30 & -.38 & .17 \\
\hline PID Submissiveness & .66 & .00 & -.03 & -.13 & .02 & .46 & .01 & .00 & -.01 & .04 & .67 \\
\hline PID Suspiciousness &.$\overline{55}$ & .30 & .26 & .26 & .22 & .61 & .29 & .25 & .18 & .19 & $-\overline{04}$ \\
\hline DAPP Suspiciousness &.$\overline{54}$ & .36 & 24 & .29 & .25 &. .60 & .35 & .23 & .22 & .22 & -.04 \\
\hline DAPP Self-Harm & .47 & -.06 & .21 & .43 & .12 & .50 & -.06 & .21 & .39 & .09 & .03 \\
\hline PID Manipulativeness &.$- \overline{05}$ & .81 & .00 & .12 & -.05 &.$- \overline{05}$ & .82 & .01 & .12 & -.04 & .02 \\
\hline PID Deceitfulness & .20 & .77 & .10 & .11 & -.11 & .15 & .77 & .11 & .14 & -.10 & .19 \\
\hline DAPP Callousness & .13 & .76 & .33 & .17 & .00 & .18 & .76 & .32 & .12 & -.01 & -.09 \\
\hline PID Attention Seeking & .26 & .70 & -.26 & .17 & .04 & .23 & .70 & -.25 & .20 & .04 & .20 \\
\hline DAPP Rejection & .08 &.$\overline{74}$ & -.01 & .23 & .21 & .19 &.$\overline{73}$ & -.03 & .14 & .19 & -.25 \\
\hline DAPP Narcissism & .52 & $\underline{.60}$ & -.23 & .08 & .16 & .48 & $\underline{.60}$ & -.22 & .09 & .16 & .26 \\
\hline PID Grandiosity & -.04 & .62 & .03 & .12 & .39 & -.04 & .62 & .03 & .16 & .39 & .06 \\
\hline PID Callousness & .02 & $\underline{.63}$ & .44 & .26 & -.06 & .11 & $\underline{.63}$ & .43 & .20 & -.08 & -.20 \\
\hline DAPP Intimacy Problems & .00 &.$- \overline{06}$ & .80 & .07 & -.04 & .04 &.$- \overline{06}$ & .80 & .05 & -.04 & -.10 \\
\hline PID Intimacy Avoidance & -.07 & .00 & .74 & .13 & -.04 & -.04 & .00 & .74 & .13 & -.05 & -.08 \\
\hline DAPP Restricted Expression & .25 & .04 & .74 & -.07 & .06 & .21 & .04 & .74 & -.04 & .07 & .17 \\
\hline PID Withdrawal & .23 & .02 & .80 & .10 & .04 & .26 & .01 &.$\overline{79}$ & .07 & .03 & -.03 \\
\hline PID Restricted Affectivity & .01 & .29 & .69 & -.04 & -.01 & -.01 & .29 & .70 & -.01 & -.01 & .08 \\
\hline DAPP Stimulus Seeking & .11 & .40 &.$- \overline{16}$ & .69 & -.24 & .18 & .41 &.$- \overline{17}$ & .66 & -.26 & -.10 \\
\hline PID Risk Taking & -.18 & .32 & -.11 & .64 & -.17 & -.12 & .34 & -.11 & .63 & -.18 & -.13 \\
\hline PID Impulsivity & .28 & .30 & -.07 & .60 & -.27 & .36 & .31 & -.09 &.$\overline{52}$ & -.31 & -.15 \\
\hline PID Irresponsibility & .38 & .34 & .23 & .39 & $\underline{-.49}$ & .38 & .34 & .23 & .37 & $\underline{-.50}$ & .11 \\
\hline DAPP Conduct Problems & .15 & .42 & 21 & .55 & -.12 & .24 & .43 & .19 & .48 & -.15 & -.18 \\
\hline PID Unusual Beliefs \& Experiences & .27 & .18 & .15 & .64 & .31 & .26 & .20 & .16 & .70 & .30 & .17 \\
\hline PID Perceptual Dysregulation & .52 & 15 & .23 & .58 & .16 & .49 & .16 & .23 & .61 & .14 & .24 \\
\hline DAPP Cognitive Distortion & .63 & .08 & .25 & .54 & .10 & .62 & .09 & .25 &.$\overline{55}$ & .08 & .21 \\
\hline PID Eccentricity & .42 & .21 & .39 & .48 & .15 & .43 & .21 & .39 & .48 & .14 & .12 \\
\hline PID Rigid Perfectionism & .30 & .20 & .06 & $\overline{.05}$ & .74 & .34 & .19 & .06 & $\overline{.04}$ & .73 & .02 \\
\hline DAPP Compulsivity & .13 & .07 & -.02 & -.04 &. .85 & .14 & .07 & -.02 & -.01 &. .85 & .05 \\
\hline PID Hostility & .29 & .53 & .23 & .40 &.$\overline{.06}$ & .46 & .52 & .20 & .24 & $\overline{.02}$ & -.40 \\
\hline Congruence with promax rotation & .97 & .94 & .97 & .92 & 1.00 & .96 & .94 & .97 & .91 & 1.00 & .98 \\
\hline $\begin{array}{l}\text { Congruence with van den Broeck et } \\
\text { al. (2014) }\end{array}$ & .94 & .91 & .85 & .80 & .64 & -- & -- & -- & -- & -- & -- \\
\hline
\end{tabular}

Note. Factor loadings $\geq .30$ are shown in bold type. The largest loading of each facet is underlined. 
Supplemental Table S4 (Cont.). Promax-Rotated One- to Seven-Factor

Solutions for the PID-5 and the DAPP-BQ.

\begin{tabular}{|c|c|c|c|c|c|c|c|}
\hline & \multicolumn{7}{|c|}{7 Factors } \\
\hline & 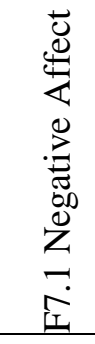 & 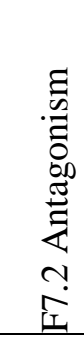 & 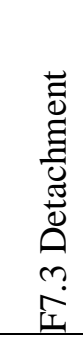 & 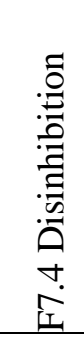 & 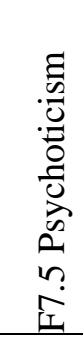 & 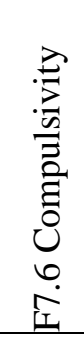 & 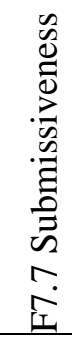 \\
\hline DAPP Anxiousness & .86 & .03 & .08 & .16 & .07 & .19 & .18 \\
\hline PID Anxiousness &.$\overline{85}$ & .05 & -.01 & .06 & .09 & .20 & .03 \\
\hline DAPP Identity Problems & .82 & .10 & .35 & .04 & .13 & -.03 & .02 \\
\hline PID Depressivity &.$\overline{78}$ & .06 & .31 & .09 & .25 & -.05 & .09 \\
\hline PID Anhedonia &.$\underline{.68}$ & .05 & .48 & -.06 & .02 & -.13 & .04 \\
\hline DAPP Insecure Attachment & .69 & .22 & -.38 & .00 & .24 & .02 & .06 \\
\hline DAPP Submissiveness & .62 & -.01 & .03 & .05 & .16 & .03 & .59 \\
\hline PID Separation Insecurity & .66 & .22 & -.36 & -.03 & .15 & -.06 & .13 \\
\hline DAPP Affective Lability &.$\overline{.73}$ & .18 & -.04 & .38 & .19 & .25 & -.17 \\
\hline DAPP Social Avoidance &.$\underline{.66}$ & .09 & .40 & -.08 & .17 & .05 & .19 \\
\hline PID Emotional Lability & .67 & .08 & -.14 & .36 & .22 & .24 & -.05 \\
\hline PID Perseveration & .64 & .16 & .21 & .28 & .04 & .29 & .20 \\
\hline DAPP Oppositionality &.$\overline{.59}$ & .12 & .18 & .45 & -.03 & -.25 & .25 \\
\hline PID Distractibility & .57 & .07 & .20 & .45 & .06 & -.19 & .24 \\
\hline PID Submissiveness & .45 & .02 & -.02 & -.10 & .15 & .04 & .66 \\
\hline PID Suspiciousness & .58 & .34 & .15 & -.08 & .47 & .03 & $-\overline{13}$ \\
\hline DAPP Suspiciousness &.$\overline{.57}$ & .39 & .14 & -.03 & .47 & .08 & -.13 \\
\hline DAPP Self-Harm & $\overline{.46}$ & -.04 & .16 & .17 & .47 & .04 & -.01 \\
\hline PID Manipulativeness & -.06 & $\underline{.80}$ & .01 & .22 & -.02 & .01 & .04 \\
\hline PID Deceitfulness & .15 & .77 & .09 & .17 & .09 & -.08 & .19 \\
\hline DAPP Callousness & .18 & .77 & .29 & .12 & .13 & -.03 & -.11 \\
\hline PID Attention Seeking & .19 &.$\overline{.68}$ & -.25 & .25 & .07 & .12 & .22 \\
\hline DAPP Rejection & .16 & .70 & -.02 & .24 & -.01 & .29 & -.22 \\
\hline DAPP Narcissism & .45 & .59 & -.22 & .13 & .06 & .25 & .28 \\
\hline PID Grandiosity & -.09 & .63 & .01 & .03 & .20 & .36 & .03 \\
\hline PID Callousness & .10 &.$\overline{.64}$ & .38 & .14 & .22 & -.14 & -.23 \\
\hline DAPP Intimacy Problems & .05 & -.06 & .81 & .04 & .04 & -.01 & -.09 \\
\hline PID Intimacy Avoidance & -.04 & -.01 & .76 & .12 & .05 & .01 & -.06 \\
\hline DAPP Restricted Expression & .22 & .06 & .74 & -.10 & .08 & .08 & .16 \\
\hline PID Withdrawal & .27 & .04 &.$\overline{.76}$ & -.04 & .21 & -.01 & -.05 \\
\hline PID Restricted Affectivity & .00 & .31 & .69 & -.06 & .07 & -.03 & .06 \\
\hline DAPP Stimulus Seeking & .13 & .34 & -.12 & .76 & .19 & -.06 & -.01 \\
\hline PID Risk Taking & -.17 & .27 & -.07 & .67 & .20 & -.03 & -.07 \\
\hline PID Impulsivity & .33 & .24 & -.05 & .67 & .10 & -.10 & -.06 \\
\hline PID Irresponsibility & .38 & .31 & .25 &.$\underline{.51}$ & .10 & -.36 & .17 \\
\hline DAPP Conduct Problems & .21 & .40 & .19 & .47 & .27 & -.09 & -.16 \\
\hline PID Unusual Beliefs \& Experiences & .17 & .21 & .08 & .28 & .76 & .19 & .10 \\
\hline PID Perceptual Dysregulation & .42 & .16 & .18 & .32 & .64 & .11 & .20 \\
\hline DAPP Cognitive Distortion & .56 & .09 & .20 & .29 &.$\overline{.57}$ & .07 & .18 \\
\hline PID Eccentricity & .38 & .22 & .36 & .27 & .49 & .13 & .10 \\
\hline PID Rigid Perfectionism & .28 & .18 & .08 & -.04 & .07 & .83 & .03 \\
\hline DAPP Compulsivity & .08 & .08 & -.02 & -.22 & .17 &.$\overline{84}$ & .02 \\
\hline PID Hostility & .44 & .50 & .18 & .29 & .13 & .08 & -.38 \\
\hline Congruence with promax rotation & .97 & .93 & .97 & .95 & .89 & .98 & .97 \\
\hline $\begin{array}{l}\text { Congruence with van den Broeck et } \\
\text { al. (2014) }\end{array}$ & -- & -- & -- & -- & -- & -- & -- \\
\hline
\end{tabular}

Note. Factor loadings $\geq .30$ are shown in bold type. The largest loading of each facet is underlined. 
Supplemental Table S5. Parallel Analysis and Velicer's MAP for the Joint Factor Analysis of the PID-5 and the DAPP-BQ.

\begin{tabular}{|c|c|c|c|c|c|}
\hline & \multicolumn{2}{|c|}{ Parallel } & \multirow{2}{*}{$\begin{array}{c}\text { Velicer's Average } \\
\text { Squared Correlations } \\
\text { (MAP) }\end{array}$} \\
\hline & Eigenvalues & explained & Means & Percentile & \\
\hline 1 & 15.006 & 34.898 & 1.676639 & 1.752725 & .133008 \\
\hline 2 & 4.620 & 10.743 & 1.602973 & 1.656936 & .046770 \\
\hline 3 & 4.112 & 9.563 & 1.546660 & 1.593974 & .036857 \\
\hline 4 & 2.744 & 6.382 & 1.499081 & 1.540998 & .024118 \\
\hline 5 & 1.676 & 3.898 & 1.455687 & 1.493917 & .020849 \\
\hline 6 & 1.325 & 3.082 & 1.415693 & 1.449693 & .019348 \\
\hline 7 & 1.081 & 2.514 & 1.379660 & 1.412893 & .018084 \\
\hline 8 & .998 & 2.321 & 1.344780 & 1.379732 & .017861 \\
\hline 9 & .903 & 2.100 & 1.312683 & 1.342668 & .018036 \\
\hline 10 & .891 & 2.071 & 1.279926 & 1.307794 & .018287 \\
\hline 11 & .783 & 1.821 & 1.250037 & 1.276479 & .017796 \\
\hline 12 & .695 & 1.616 & 1.221117 & 1.248405 & .017751 \\
\hline 13 & .575 & 1.337 & 1.192763 & 1.219266 & .017978 \\
\hline 14 & .540 & 1.257 & 1.165618 & 1.191269 & .018736 \\
\hline 15 & .504 & 1.173 & 1.138811 & 1.162823 & .019586 \\
\hline 16 & .500 & 1.162 & 1.112789 & 1.137622 & .020751 \\
\hline 17 & .466 & 1.085 & 1.087141 & 1.111046 & .021219 \\
\hline 18 & .430 & 1.001 & 1.062585 & 1.086532 & .023069 \\
\hline 19 & .396 & .921 & 1.037885 & 1.060133 & .024447 \\
\hline 20 & .363 & .844 & 1.013966 & 1.035766 & .025970 \\
\hline 21 & .356 & .829 & .991116 & 1.013352 & .028055 \\
\hline 22 & .320 & .744 & .968423 & .990002 & .030719 \\
\hline 23 & .318 & .739 & .945351 & .968039 & .032821 \\
\hline 24 & .307 & .715 & .922702 & .944704 & .036143 \\
\hline 25 & .282 & .655 & .900750 & .921518 & .038675 \\
\hline 26 & .260 & .605 & .878822 & .901456 & .042490 \\
\hline 27 & .249 & .578 & .857066 & .878873 & .046690 \\
\hline 28 & .239 & .555 & .835114 & .857191 & .051706 \\
\hline 29 & .212 & .494 & .813327 & .834688 & .057021 \\
\hline 30 & .188 & .438 & .791777 & .812630 & .063181 \\
\hline 31 & .186 & .433 & .770730 & .792750 & .068066 \\
\hline 32 & .168 & .390 & .749776 & .770945 & .076368 \\
\hline 33 & .161 & .375 & .728650 & .749405 & .087085 \\
\hline 34 & .148 & .344 & .707871 & .729694 & .097334 \\
\hline 35 & .140 & .325 & .686089 & .707815 & .115957 \\
\hline 36 & .136 & .316 & .664056 & .686842 & 130570 \\
\hline 37 & .128 & .297 & .642661 & .664567 & .152214 \\
\hline 38 & .119 & .278 & .621171 & .642785 & .168034 \\
\hline 39 & .109 & .253 & .598198 & .620313 & .209620 \\
\hline 40 & .103 & .240 & .574055 & .598629 & .258056 \\
\hline 41 & .098 & .228 & .548844 & .572938 & .353218 \\
\hline 42 & .086 & .200 & .520981 & .546560 & .511654 \\
\hline 43 & .078 & .182 & .485974 & .517118 & 1.000000 \\
\hline
\end{tabular}

Note. In Velicer's MAP the smallest average squared correlation was .017751 and the number of recommended components was 11 . 
Supplemental Table S6. Raw Pearson's Correlations Between the PID-5 and the DAPP-BQ Facets.

DAPP-BQ Facets

$\underline{\text { PID-5 Facets }}$

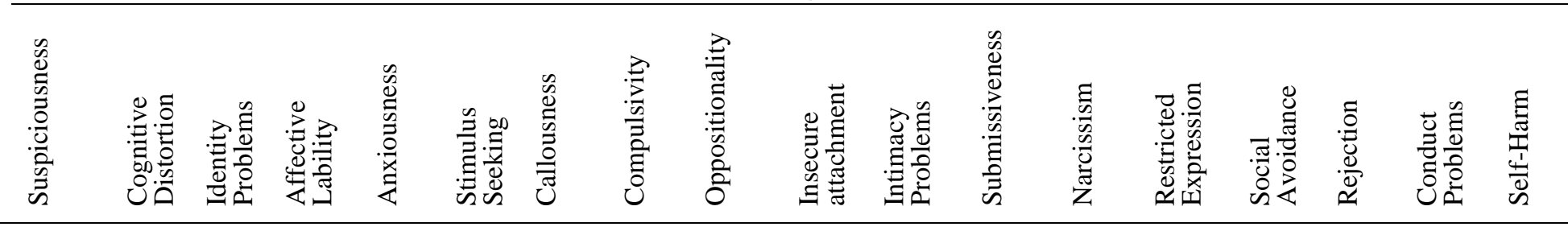

\begin{tabular}{|c|c|c|c|c|c|c|c|c|c|c|c|c|c|c|c|c|c|c|}
\hline Suspiciousness & .84 & .55 & .58 & .54 & .53 & .26 & .46 & .19 & .34 & .46 & .19 & .41 & .43 & .31 & .53 & .32 & .32 & .39 \\
\hline Perceptual Dysregulation & $\overline{.55}$ & .84 & .54 & .57 & .51 & .39 & .35 & .15 & .48 & .41 & .16 & .44 & .41 & .26 & .49 & .26 & .39 & .51 \\
\hline Anhedonia & .41 & .49 & .84 & .42 & .60 & .03 & .27 & -.04 & .51 & .29 & .38 & .41 & .18 & .48 & .58 & .09 & .23 & .42 \\
\hline Depressivity & .55 & .67 & .85 & .59 & .75 & .21 & .28 & .06 & .58 & .49 & .26 & .56 & .35 & .40 & .66 & .16 & .39 & .65 \\
\hline Emotional Lability & .48 & .57 & $\overline{.52}$ & .84 & $\overline{.65}$ & .39 & .23 & .19 & .44 & .57 & -.01 & .45 & .48 & .06 & .42 & .28 & .29 & .42 \\
\hline Anxiousness & .58 & .55 & .67 & .67 & .82 & .18 & .17 & .21 & .43 & .60 & .05 & .54 & .47 & .20 & .53 & .21 & .24 & .41 \\
\hline Risk Taking & .15 & .13 & -.01 & .17 & .00 & .77 & .24 & -.10 & .14 & .04 & -.07 & -.05 & .18 & -.02 & -.11 & .29 & .48 & .11 \\
\hline Callousness & .44 & .32 & .29 & .27 & .11 & .32 & .72 & -.01 & .23 & .11 & .30 & -.01 & .22 & .24 & .22 & .47 & .52 & .18 \\
\hline Rigid Perfectionism & .33 & .27 & .25 & .38 & .37 & .02 & .19 & .77 & .01 & .25 & .02 & .20 & .35 & .20 & .26 & .33 & .10 & .19 \\
\hline Irresponsibility & .35 & .48 & .42 & .39 & .36 & .49 & .43 & $\overline{-.29}$ & .68 & .27 & .20 & .34 & .29 & .19 & .36 & .25 & .47 & .29 \\
\hline Distractibility & .38 & .60 & .52 & .50 & .56 & .37 & .24 & -.17 & .73 & .36 & .13 & .46 & .30 & .23 & .44 & .16 & .35 & .31 \\
\hline Separation Insecurity & .41 & .38 & .43 & .47 & .50 & .28 & .15 & .08 & $\overline{.33}$ & .75 & -.25 & .43 & .51 & -.02 & .37 & .20 & .15 & .29 \\
\hline Intimacy Avoidance & .12 & .16 & .23 & .04 & .05 & -.01 & .24 & -.06 & .10 & -.27 & .70 & .00 & -.13 & .44 & .22 & .00 & .18 & .17 \\
\hline Submissiveness & .28 & .37 & .39 & .27 & .49 & .03 & .02 & .14 & .26 & .36 & $\overline{.01}$ & .71 & .36 & .16 & .39 & -.09 & .00 & .24 \\
\hline Attention Seeking & .31 & .29 & .19 & .40 & .29 & .45 & .43 & .08 & .25 & .38 & -.16 & .20 & .73 & -.11 & .11 & .55 & .34 & .15 \\
\hline Restricted Affectivity & .21 & .20 & .29 & -.02 & .08 & .07 & .39 & .01 & .12 & -.10 & .43 & .03 & $\overline{.01}$ & .65 & .30 & .19 & .23 & .07 \\
\hline Perseveration & .47 & .59 & .60 & .58 & .67 & .27 & .33 & .22 & .54 & .44 & .13 & .51 & .42 & .29 & .54 & .32 & .27 & .36 \\
\hline Unusual Beliefs \& Experiences & .53 & .67 & .30 & .46 & .33 & .37 & .31 & .21 & .26 & .31 & .09 & .27 & .34 & .18 & .27 & .30 & .39 & .40 \\
\hline Deceitfulness & .38 & .30 & .27 & .30 & .21 & .36 & .63 & .02 & .28 & .27 & .06 & .22 & .48 & .13 & .19 & .43 & .43 & .16 \\
\hline Impulsivity & .31 & .42 & .28 & .53 & .33 & 67 & .34 & -.15 & .43 & .34 & .06 & .24 & .33 & .00 & .16 & .33 & .43 & .25 \\
\hline Hostility & .55 & .43 & .46 & .66 & .38 & .40 & .55 & .05 & .33 & .32 & .19 & .12 & .40 & .16 & .36 & .56 & .53 & .27 \\
\hline Eccentricity & .51 & .67 & .52 & .54 & .45 & .29 & .37 & .12 & .41 & .30 & .29 & .34 & .32 & .35 & .51 & .29 & .42 & .45 \\
\hline Manipulativeness & .24 & .13 & .08 & .22 & .02 & .38 & .59 & .03 & .16 & .14 & -.02 & -.04 & .40 & .00 & -.03 & .53 & .37 & .04 \\
\hline Grandiosity & .28 & .17 & .01 & .21 & .04 & .24 & .43 & .27 & .00 & .14 & -.06 & -.03 & .46 & .05 & .09 & .54 & .21 & .06 \\
\hline Withdrawal & .37 & .38 & .47 & .21 & .25 & -.02 & .28 & .04 & .23 & .00 & .61 & .18 & -.04 & .60 & .61 & .05 & .25 & .27 \\
\hline
\end{tabular}

Note. Correlations over .70 are in boldtype and underlined. Correlations are significant at $p<.01$ from $r \geq .10$ and at $p<.001$ from $r \geq .13$. 
Supplemental Table S7. Multitrait-Multimethod Matrix for the Disattenuated $\left(r_{c}\right)$ and Raw $(r)$ Pearson's Correlations Between the PID-5 and the DAPP-BQ Domains, with Cronbach's Alphas $(\alpha)$ in the Diagonal.

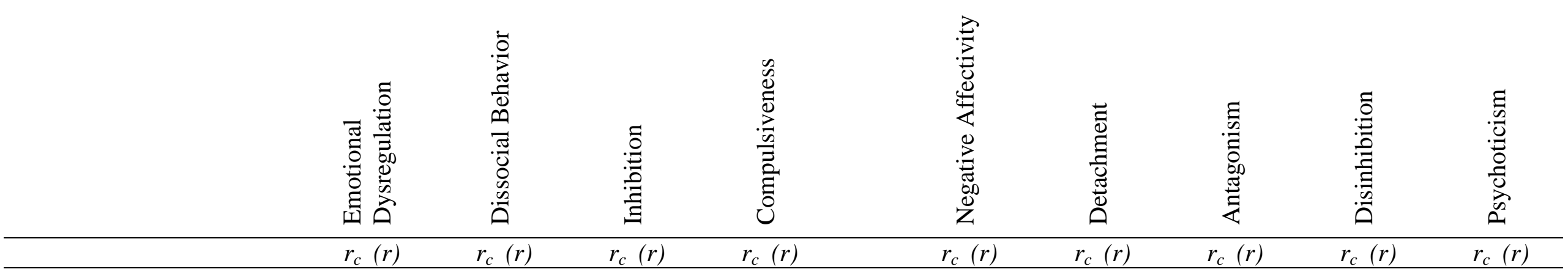

\section{DAPP-BQ}

Emotional Dysregulation

Dissocial Behavior

Inhibition

Compulsiveness

PID-5

Negative Affectivity

Detachment

Antagonism

Disinhibition

Psychoticism

$$
\begin{aligned}
& \alpha=.98 \\
& .53(.51) \quad \alpha=.94 \\
& .28(.26) \quad .10(.09) \quad \alpha=.90 \\
& \text {-.15 (-.14) } \quad-.11(-.10) \quad-.07 \quad(-.06) \quad \alpha=.91
\end{aligned}
$$

\begin{tabular}{|c|c|c|c|}
\hline $.87(.83)$ & .44 (.41) & $.00(.00)$ & $-.03(-.03)$ \\
\hline .47 (.45) & .19 (.18) & $.85(.77)$ & $-.17(-.16)$ \\
\hline .28 (.26) & .75 (.69) & $.04(.04)$ & $.03(.03)$ \\
\hline .66 (.63) & $.62(.58)$ & $.20(.17)$ & $-.52(-.48)$ \\
\hline $.68(.66)$ & $.54(.51)$ & $.32(.30)$ & $.03(-.03)$ \\
\hline
\end{tabular}

Cronbach's $\alpha$ (reliability)

Monotrait-heteromethod (validity)

Heterotrait-monomethod

Heterotrait-heteromethod

Note. Significant coefficients $(p<.05)$ are in boldtype. As can be seen, the monotrait-heteromethod "diagonal” indicating validity is not a diagonal at all, as there is not a biunivocal correspondence between PID-5 and DAPP-BQ domains. 
Supplemental Table S8. Regression Coefficients of DAPP-BQ Facets Predicting the PID-5.

\begin{tabular}{|c|c|c|}
\hline 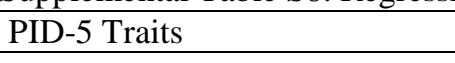 & Adj. $R^{2}$ & Standardized $\beta$ for DAPP-BQ Traits \\
\hline Depressivity & .78 & Identity Problems (.71), Self-Harm (.24), Anxiousness (.21) \\
\hline Anhedonia & .73 & Identity Problems (.90), Affective Lability (-.15), Intimacy Problems (.10) \\
\hline Suspiciousness & .72 & Suspiciousness (.77), Social Avoidance (.13) \\
\hline Emotional Lability & .72 & Affective Lability (.77), Insecure Attachment (.13) \\
\hline Perceptual Dysregulation & .71 & Cognitive Distortion (.80), Stimulus Seeking (.11) \\
\hline Anxiousness & .70 & Anxiousness (.65), Insecure Attachment (.16), Suspiciousness (.13) \\
\hline Rigid Perfectionism & .65 & Compulsivity (.71), Affective Lability (.23), Restricted Expression (.11) \\
\hline Risk Taking & .63 & Stimulus Seeking (.74), Anxiousness (-.22), Conduct Problems (.14) \\
\hline Withdrawal & .62 & Social Avoidance (.55), Intimacy Problems (.39), Narcissism (-.24), Conduct Problems (.15) \\
\hline Hostility & .62 & Affective Lability (.52), Callousness (.23), Submissiveness. (-.23), Suspiciousness (.17), Conduct Problems (.16) \\
\hline Attention Seeking & .60 & Narcissism (.69), Social Avoidance (-.21), Stimulus Seeking (.14), Callousness (.14) \\
\hline Distractibility & .58 & Oppositionality (.58), Cognitive Distortion (.26) \\
\hline Separation Insecurity & .58 & Insecure Attachment (.66), Narcissism (.17) \\
\hline Callousness & .58 & Callousness (.60), Conduct Problems (.21), Intimacy Problems (.14), Submissiveness (-.13) \\
\hline Irresponsibility & .56 & Oppositionality (.51), Callousness (.20), Conduct Problems (.18), Compulsivity (-.18) \\
\hline Impulsivity & .54 & Stimulus Seeking (.51), Affective Lability (.34), Compulsivity (-.16) \\
\hline Submissiveness & .53 & Submissiveness (.74), Conduct Problems (-.14) \\
\hline Unusual Beliefs \& Experiences & .53 & Cognitive Distortion (.69), Identity Problems (-.32), Suspiciousness (.30) \\
\hline Perseveration & .52 & Anxiousness (.48), Cognitive Distortion (.24), Rejection (.17) \\
\hline Eccentricity & .50 & Cognitive Distortion (.48), Affective Lability (.22), Restricted Expression (.19) \\
\hline Restricted Affectivity & .50 & Restricted Expression (.60), Callousness (.30), Affective Lability (-.18) \\
\hline Intimacy Avoidance & .49 & Intimacy Problems (.70) \\
\hline Manipulativeness & .46 & Callousness (.48), Social Avoidance (-.27), Narcissism (.20), Rejection (.19) \\
\hline Deceitfulness & .45 & Callousness (.52), Narcissism (.26) \\
\hline Grandiosity & .37 & Narcissism (.35), Rejection (.35), Anxiousness (-.24), Compulsivity (.16) \\
\hline
\end{tabular}

Note. PID-5 traits are presented in descending order according to the percentage of variance explained by the DAPP-BQ traits (adjusted $R^{2}$ ). All $R^{2}$ and beta coefficients are significant at $p<.001$. 
Supplemental Table S9. Regression Coefficients of PID-5 Facets Predicting the DAPP-BQ.

\begin{tabular}{|c|c|c|}
\hline DAPP-BQ Traits & Adj. $R^{2}$ & Standardized $\beta$ for PID-5 Facets \\
\hline Identity Problems & .83 & Anhedonia (.47), Depressivity (.42), Emotional Lability (.15) \\
\hline Affective Lability & .82 & Emotional Lability (.58), Hostility (.31), Anxiousness (.19) \\
\hline Cognitive Distortion & .78 & Perceptual Dysregulation (.50), Depressivity (.23), Distractibility (.16), Unusual Beliefs \& Experiences (.17) \\
\hline Anxiousness & .77 & Anxiousness (.44), Depressivity (.28), Emotional Lability (.16), Perseveration (.15) \\
\hline Stimulus Seeking & .76 & Risk Taking (.61), Impulsivity (.40), Separation Insecurity (.12) \\
\hline Suspiciousness & .74 & Suspiciousness (.63), Anxiousness (.16), Unusual Beliefs \& Experiences (.14), Callousness (.11) \\
\hline Narcissism & .65 & Attention Seeking (.50), Anxiousness (.22), Grandiosity (.21), Separation Insecurity (.17) \\
\hline Insecure Attachment & .65 & Separation Insecurity (.51), Emotional Lability (.21), Anxiousness (.19), Intimacy Avoidance (-.14) \\
\hline Callousness & .65 & Callousness (.45), Manipulativeness (.19), Deceitfulness (.17), Suspiciousness (.15), Grandiosity (.09) \\
\hline Compulsivity & .64 & Rigid Perfectionism (.75), Irresponsibility (-.24) \\
\hline Oppositionality & .64 & Distractibility (.44), Irresponsibility (.32), Depressivity (.23), Withdrawal (-.12) \\
\hline Submissiveness & .63 & Submissiveness (.53), Depressivity (.21), Distractibility (.17), Callousness (-.17), Suspiciousness (.14) \\
\hline Social Avoidance & .61 & Withdrawal (.43), Depressivity (.28), Separation Insecurity (.20), Perseveration (.16), Risk Taking (-.11) \\
\hline Intimacy Problems & .58 & Intimacy Avoidance (.53), Withdrawal (.33), Grandiosity (-.12) \\
\hline Restricted Expression & .56 & Restricted Affectivity (.48), Withdrawal (.33), Depressivity (.20), Hostility (-.16) \\
\hline Rejection & .56 & Hostility (.28), Attention Seeking (.27), Grandiosity (.23), Submissiveness (-.22), Perseveration (.15), Manipulativeness (.14) \\
\hline Conduct Problems & .48 & Risk Taking (.35), Callousness (.25), Depressivity (.23), Hostility (.19) \\
\hline Self-Harm & .46 & Depressivity (.60), Unusual Beliefs \& Experiences (.20), Restricted Affectivity (-.12) \\
\hline
\end{tabular}

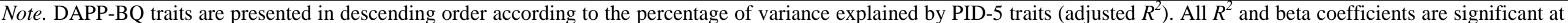
$p<.001$ except for PID Restricted Affectivity predicting DAPP Self-Harm $(p<.01)$. 
Supplemental Figure S1. Main Disattenuated $\left(r_{c}\right)$ and Raw $(r)$ Pearson's Correlations Between the PID-5 and the DAPP-BQ Traits.

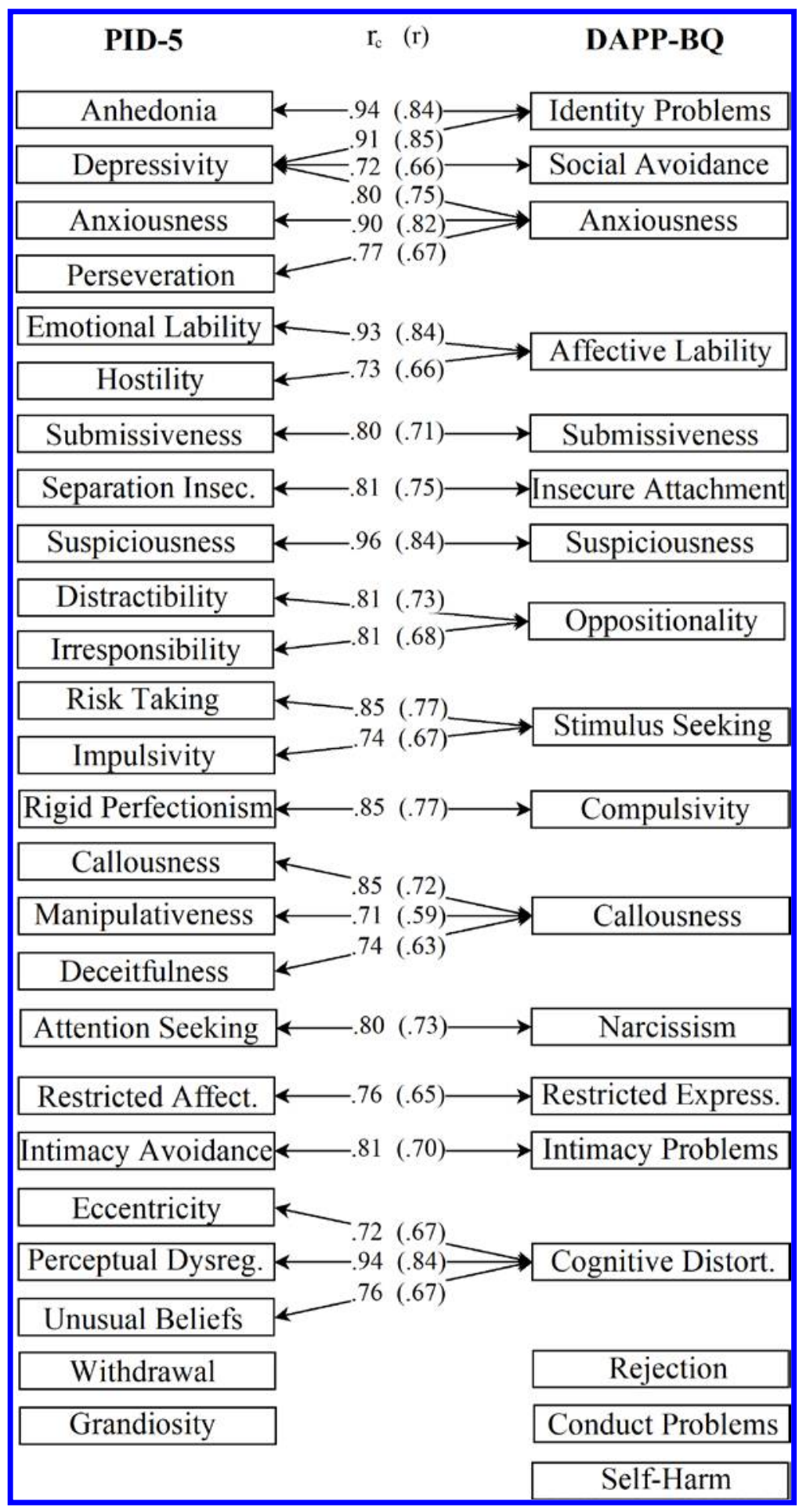

Note. All $p<.001$. 
Supplemental Figure S2. Test Information Curves From the 43 PID-5 and DAPP-BQ Facets.

\section{Negative Affect}
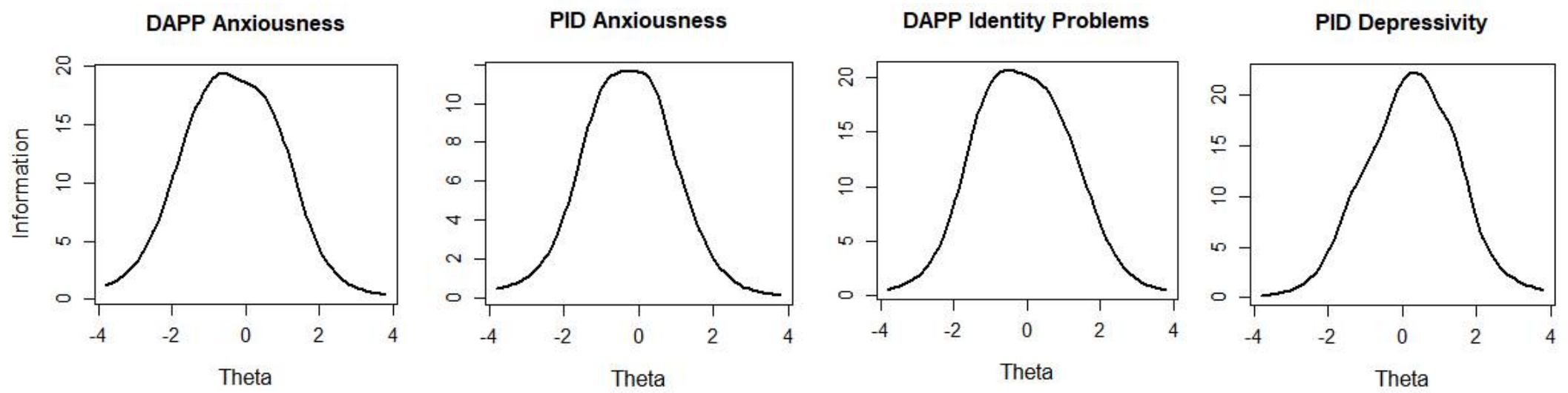

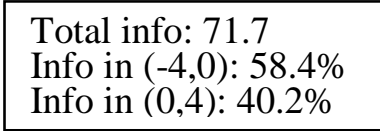

DAPP Insecure Attachment

\section{Total info: 38.2 \\ Info in $(-4,0): 57.4 \%$ \\ Info in $(0,4): 40.7 \%$}

DAPP Submissiveness

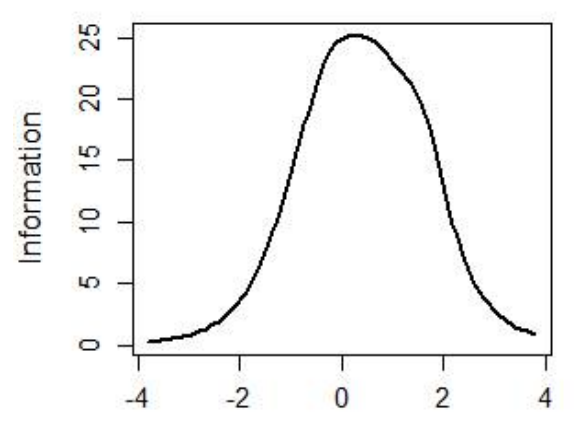

Theta

$$
\begin{aligned}
& \text { Total info: } 83.6 \\
& \text { Info in }(-4,0): 36.8 \%
\end{aligned}
$$$$
\text { Info in }(0,4): 62.4 \%
$$

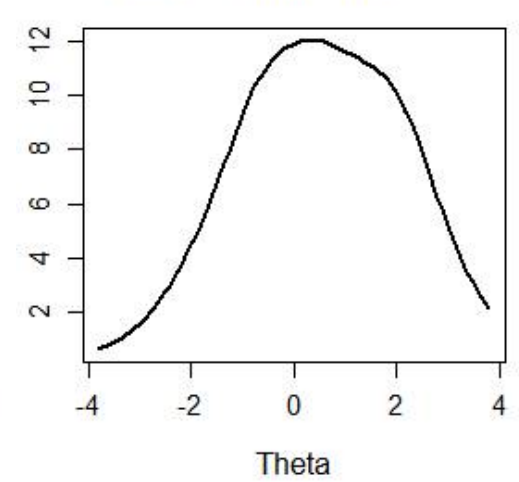

Total info: 57.4

Info in $(-4,0): 37.4 \%$

Info in $(0,4): 59.1 \%$

\section{Total info: 75.2 \\ Info in (-4,0): $53.2 \%$ \\ Info in $(0,4): 45.7 \%$}

PID Separation Insecurity

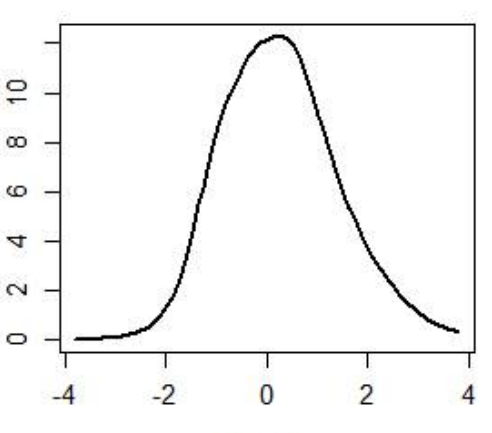

Theta

Total info: 36.4

Info in $(-4,0): 42.9 \%$

Info in $(0,4): 56.7 \%$

\section{Total info: 69.8 \\ Info in $(-4,0): 40.7 \%$ \\ Info in $(0,4): 58.6 \%$}

DAPP Affective Lability

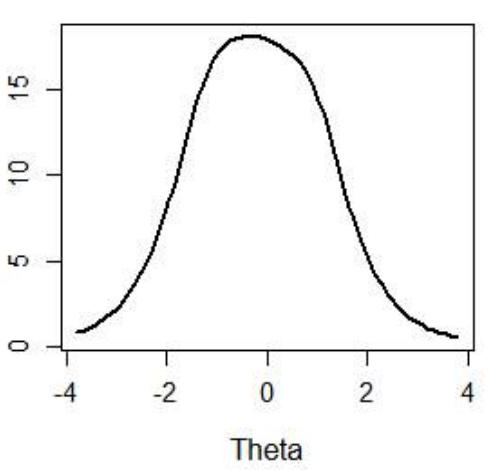

Total info: 68.0

Info in $(-4,0): 54.1 \%$

Info in $(0,4): 44.6 \%$
PID Anhedonia

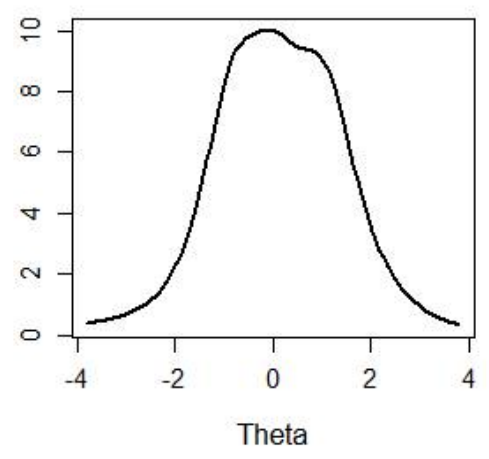

Total info: 35.4

Info in $(-4,0): 45.8 \%$

Info in $(0,4): 52.6 \%$

DAPP Social Avoidance

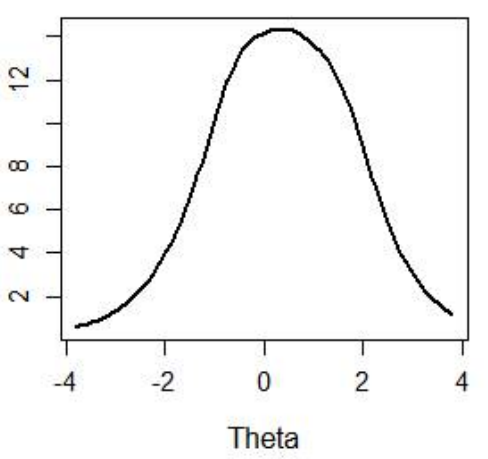

Total info: 57.3

Info in $(-4,0): 39.6 \%$

Info in $(0,4): 58.2 \%$ 


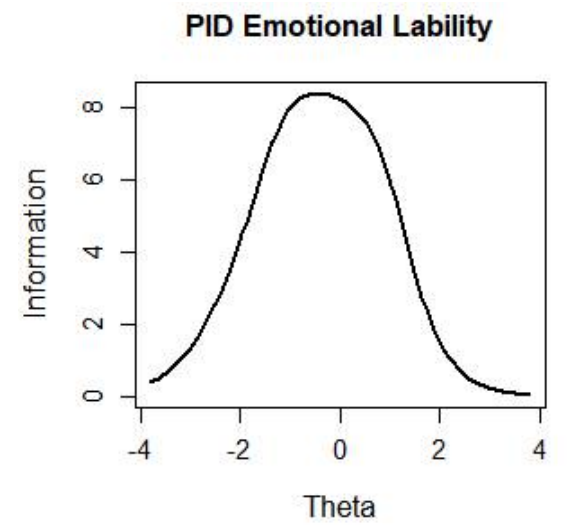

Total info: 30.0

Info in $(-4,0): 60.0 \%$

Info in $(0,4): 39.4 \%$

PID Suspiciousness

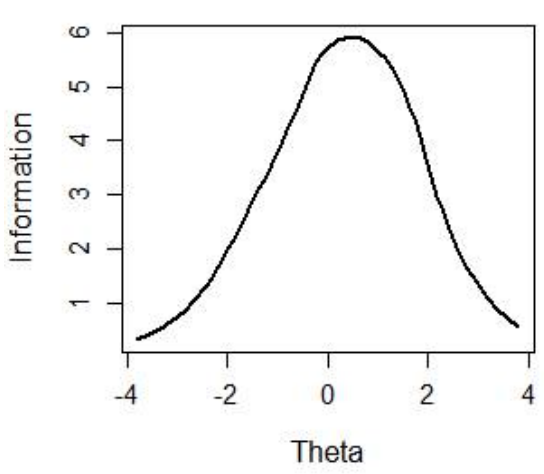

Total info: 23.8

Info in $(-4,0): 39.6 \%$

Info in $(0,4): 58.0 \%$
PID Perseveration

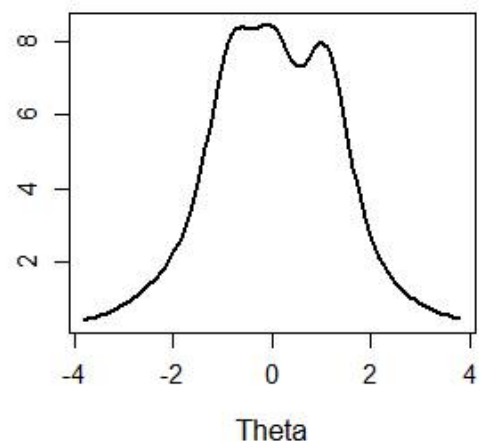

Total info: 31.1

Info in $(-4,0): 47.1 \%$

Info in $(0,4): 49.8 \%$

DAPP Suspiciousness

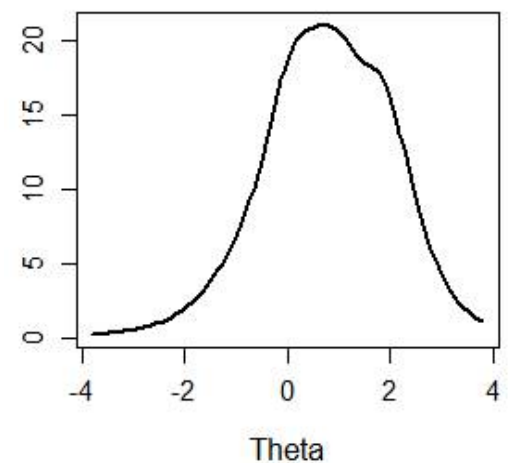

Total info: 69.5

Info in $(-4,0): 25.4 \%$

Info in $(0,4): 73.3 \%$
DAPP Oppositionality

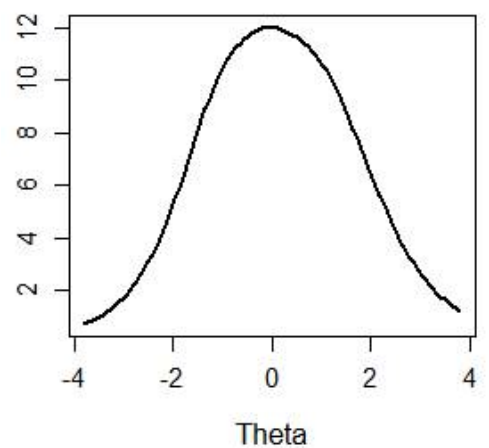

Total info: 51.9

Info in $(-4,0): 45.8 \%$

Info in $(0,4): 50.8 \%$

\section{DAPP Self-harm}

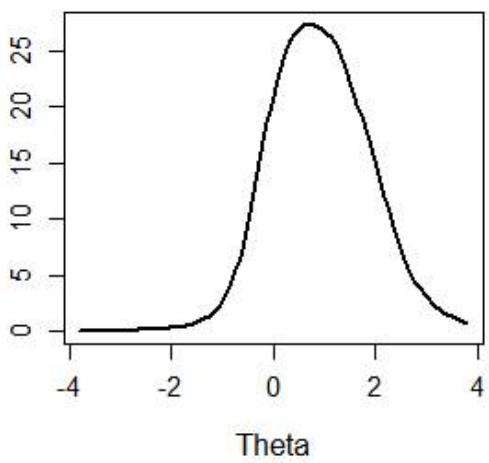

Total info: 69.3

Info in $(-4,0): 16.9 \%$

Info in $(0,4): 82.5 \%$

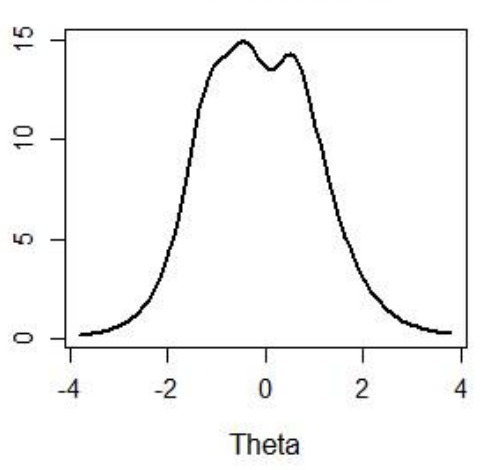

Total info: 48.0

Info in $(-4,0): 53.9 \%$

Info in $(0,4): 45.6 \%$
PID Submissiveness

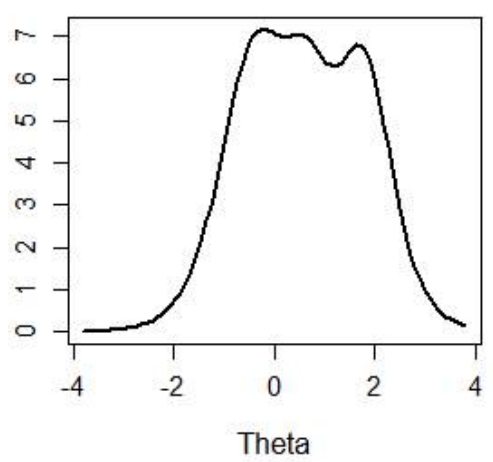

Total info: 25.9

Info in $(-4,0)$ : $34.5 \%$

Info in $(0,4): 65.4 \%$ 


\section{Antagonism}

PID Manipulativeness

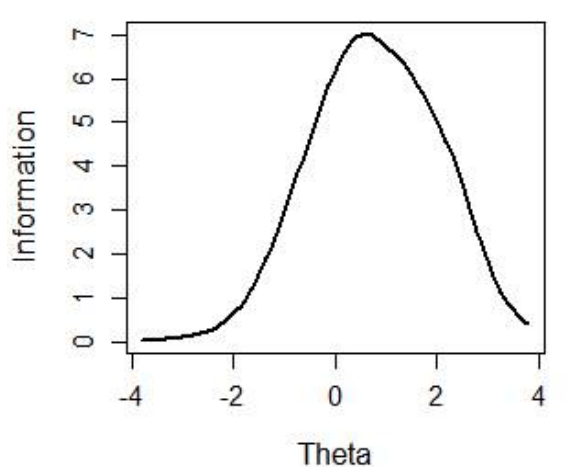

Total info: 23.9

Info in $(-4,0): 27.3 \%$

Info in $(0,4): 72.1 \%$

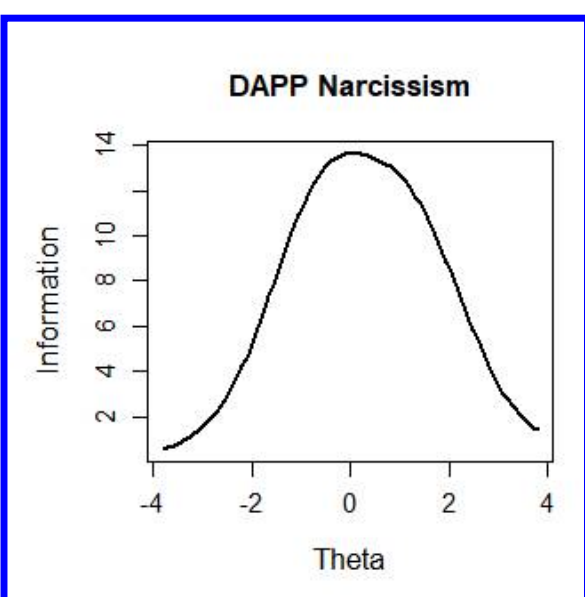

Total info: 58.8

Info in $(-4,0): 42.5 \%$

Info in $(0,4): 54.8 \%$
PID Deceitfulness

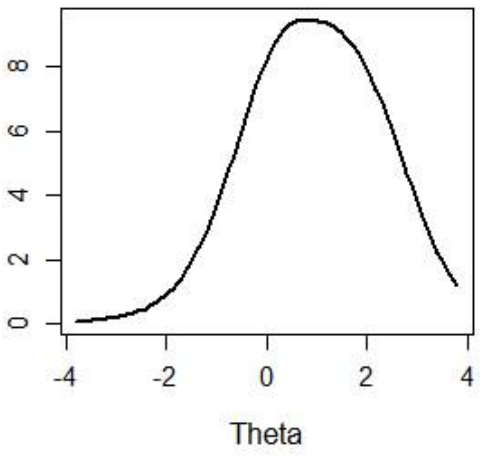

Total info: 35.7

Info in $(-4,0): 24.2 \%$

Info in $(0,4): 73.4 \%$

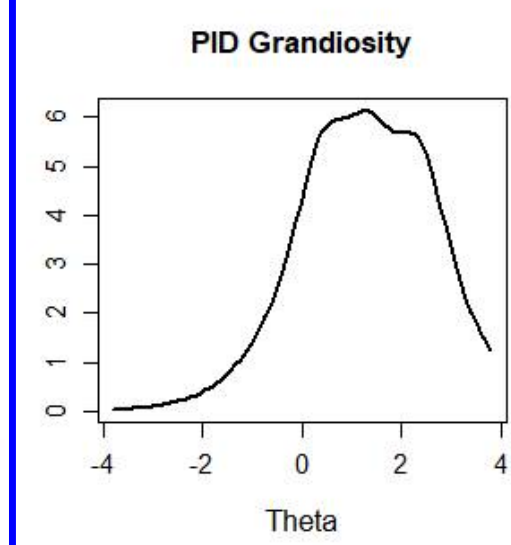

Total info: 23.0

Info in $(-4,0): 16.2 \%$

Info in $(0,4): 80.1 \%$

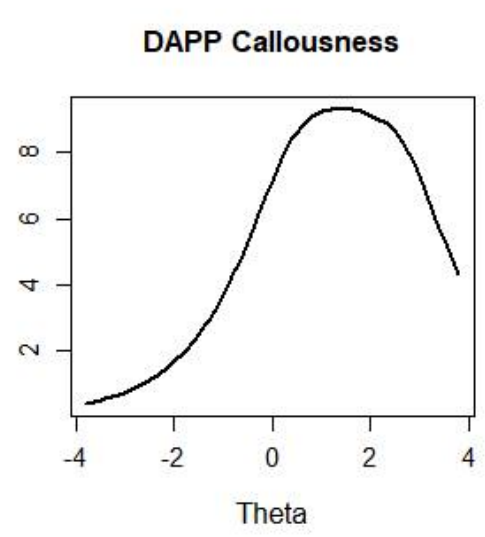

Total info: 45.6

Info in $(-4,0): 20.9 \%$

Info in $(0,4): 69.3 \%$

PID Callousness

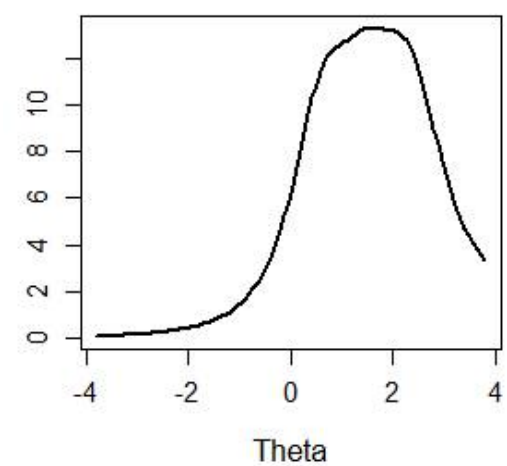

Total info: 47.4

Info in $(-4,0): 9.5 \%$

Info in $(0,4): 82.5 \%$

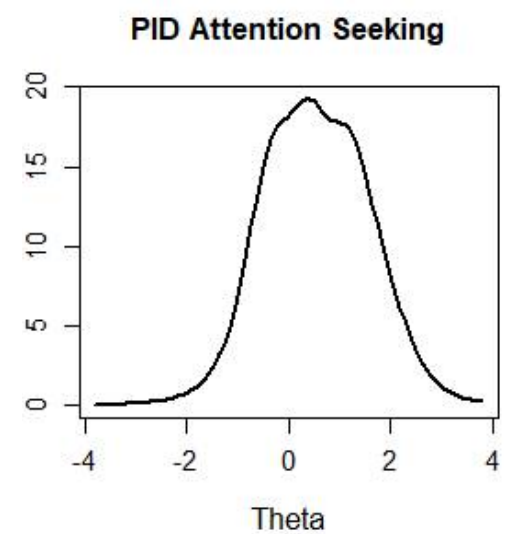

Total info: 54.5

Info in $(-4,0): 31.7 \%$

Info in $(0,4)$ : $68.1 \%$

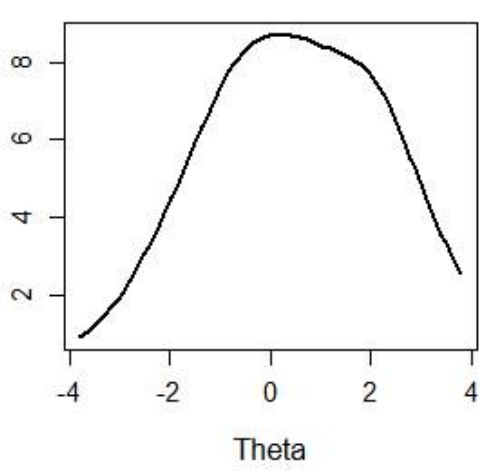

Total info: 47.7 Info in $(-4,0): 38.6 \%$ Info in $(0,4): 55.6 \%$ 


\section{Detachment}

DAPP Intimacy Problems

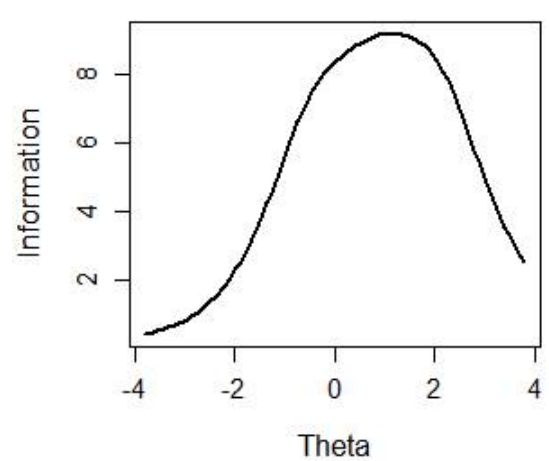

Total info: 44.2

Info in $(-4,0): 29.3 \%$

Info in $(0,4): 63.5 \%$

\section{Disinhibition}

DAPP Stimulus Seeking

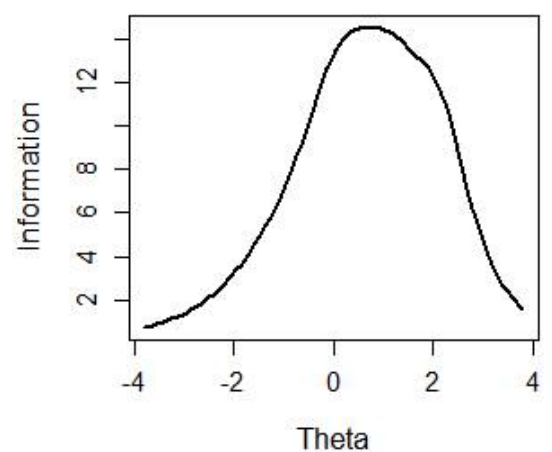

Total info: 59.3 Info in $(-4,0): 30.7 \%$ Info in $(0,4): 66.2 \%$
PID Intimacy Avoidance

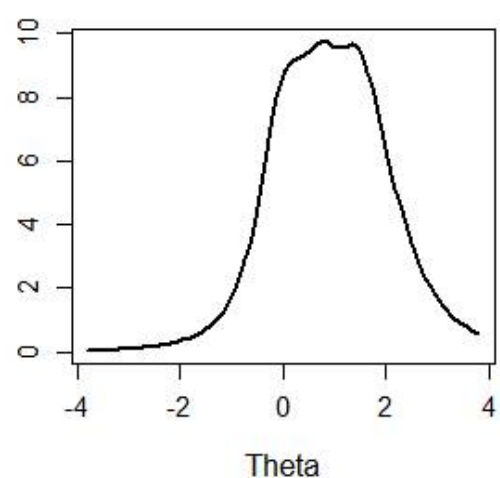

Total info: 29.1

Info in $(-4,0): 20.2 \%$

Info in $(0,4): 78.5 \%$

PID Risk Taking

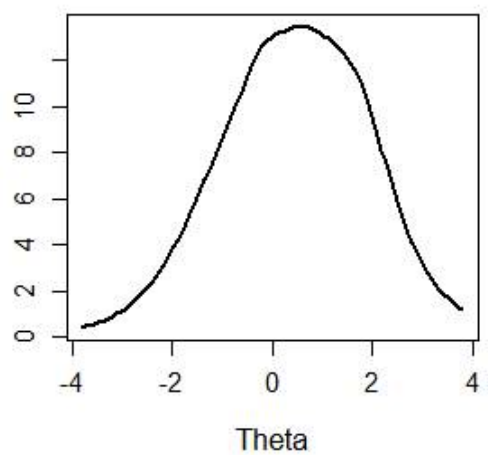

Total info: 54.1

Info in $(-4,0): 37.2 \%$

Info in $(0,4): 61.0 \%$

DAPP Restricted Expression

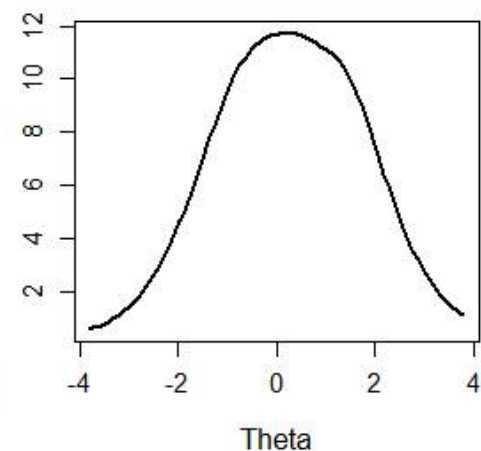

Total info: 51.4

Info in $(-4,0): 41.8 \%$

Info in $(0,4): 54.2 \%$

PID Impulsivity

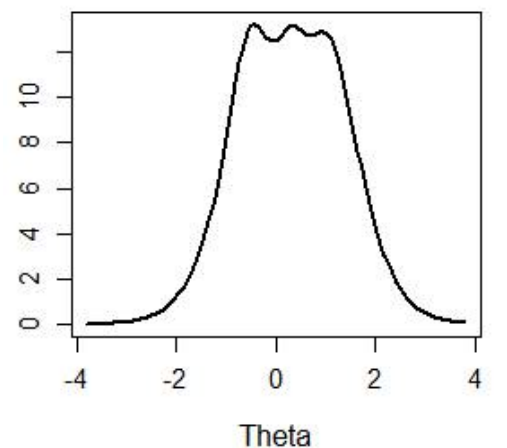

Total info: 40.3

Info in $(-4,0): 40.5 \%$

Info in $(0,4): 59.4 \%$
PID Withdrawal

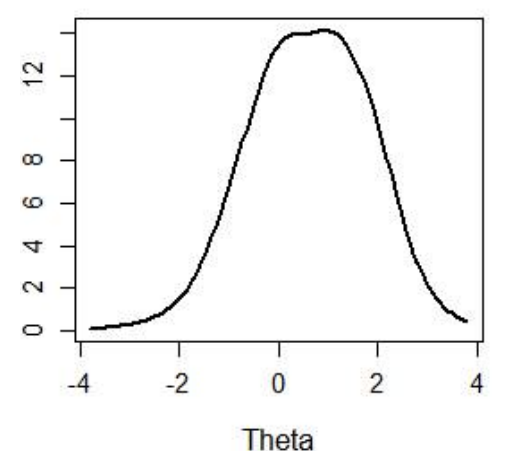

Total info: 48.3

Info in $(-4,0): 31.1 \%$

Info in $(0,4): 68.5 \%$
PID Restricted Affectivity

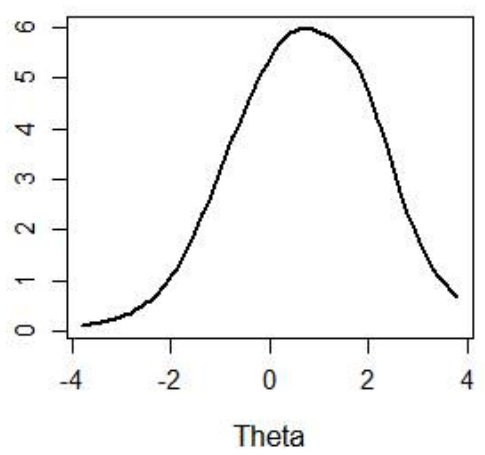

Total info: 23.3

Info in $(-4,0): 30.6 \%$

Info in $(0,4): 67.2 \%$
DAPP Conduct Problems

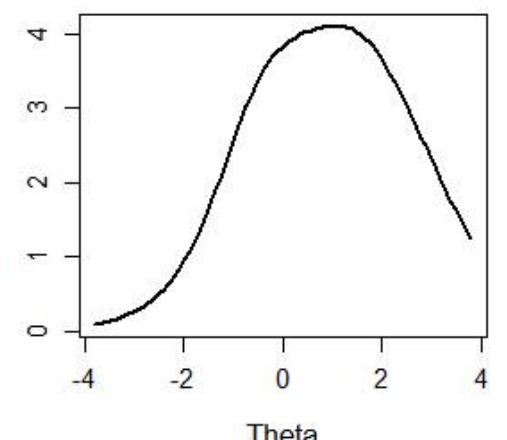

Total info: 19.2

Info in $(-4,0): 29.4 \%$ Info in $(0,4): 65.7 \%$

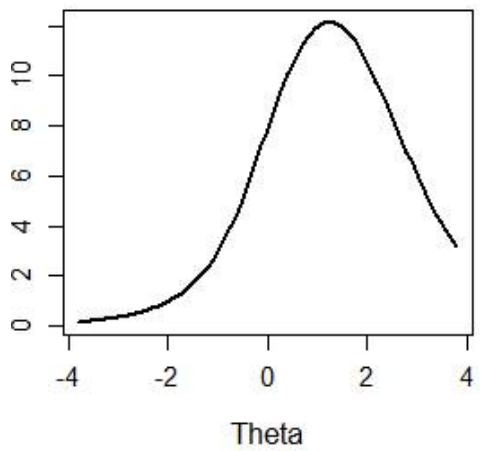

Total info: 59.3

Info in $(-4,0): 30.7 \%$ Info in $(0,4): 66.2 \%$ 


\section{Psychoticism}

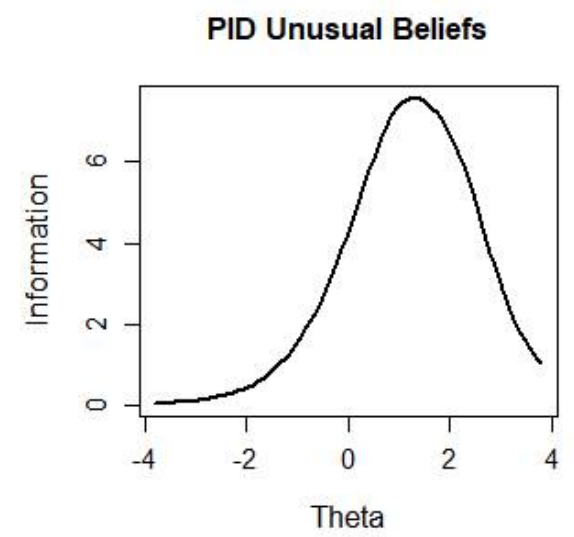

PID Perceptual Dysregulation

DAPP Cognitive Distortion

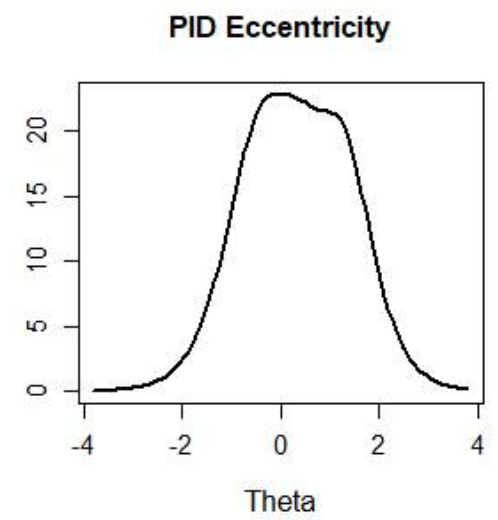

Total info: 24.6

Info in $(-4,0): 16.0 \%$

Info in $(0,4): 81.3 \%$

\section{Compulsivity}

PID Rigid Perfectionism

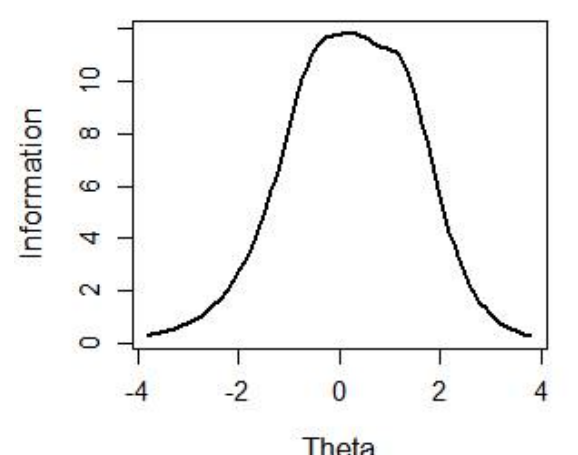

DAPP Compulsivity
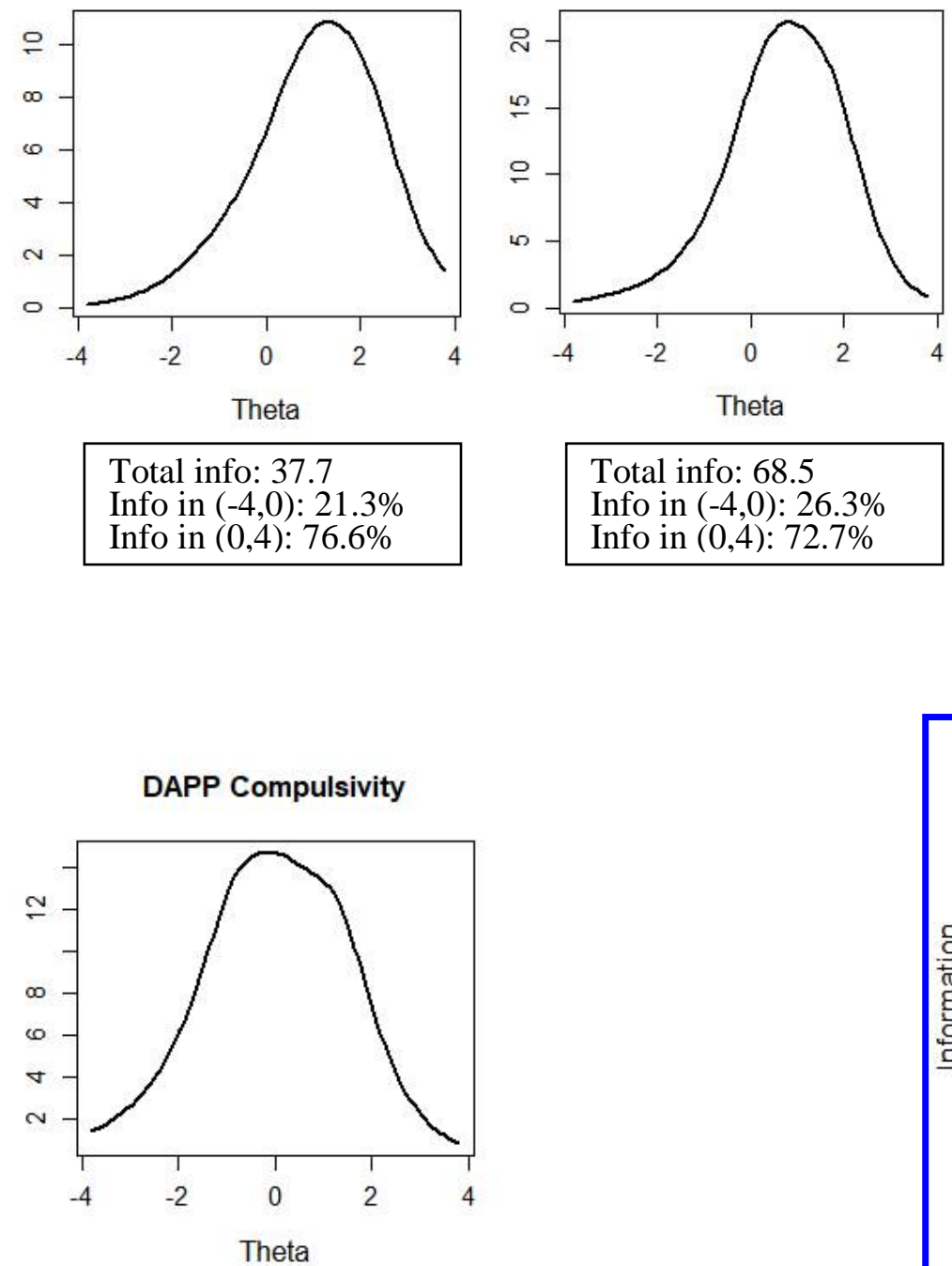

Total info: 68.5

Info in $(-4,0): 26.3 \%$

Info in $(0,4): 72.7 \%$

Total info: 71.7

Info in $(-4,0): 39.5 \%$

Info in $(0,4): 60.5 \%$

Info in $(-4,0): 21.3 \%$
Info in $(0,4): 76.6 \%$

\section{Submissiveness}

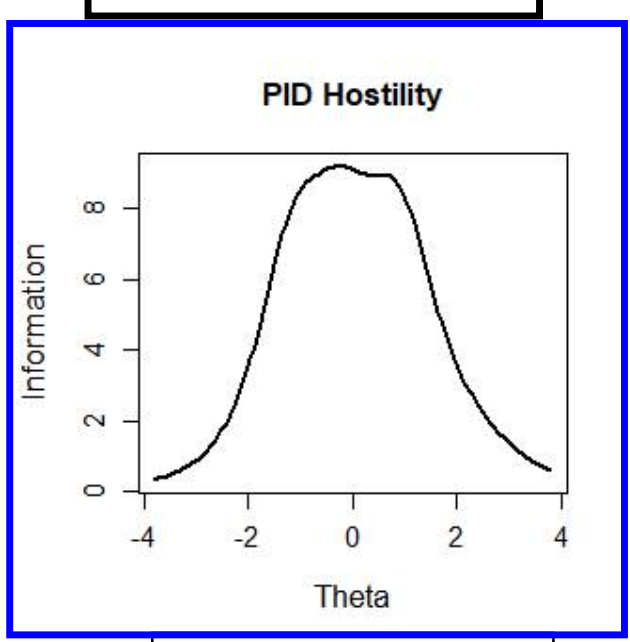

Total info: 36.4

Info in $(-4,0): 48.6 \%$

Info in $(0,4): 49.6 \%$ 
This article has been cited by:

1. Johannes Zimmermann, André Kerber, Katharina Rek, Christopher J. Hopwood, Robert F. Krueger. 2019. A Brief but Comprehensive Review of Research on the Alternative DSM-5 Model for Personality Disorders. Current Psycbiatry Reports 21:9. . [Crossref] 